\title{
Asymptotic Analysis of Ultra-relativistic Charge
}

\author{
David A. Burton, Jonathan Gratus and Robin W. Tucker \\ Department of Physics, Lancaster University \\ and The Cockcroft Institute
}

September 6, 2018

\begin{abstract}
This article offers a new approach for analysing the dynamic behaviour of distributions of charged particles in an electromagnetic field. After discussing the limitations inherent in the Lorentz-Dirac equation for a single point particle a simple model is proposed for a charged continuum interacting self-consistently with the Maxwell field in vacuo. The model is developed using intrinsic tensor field theory and exploits to the full the symmetry and light-cone structure of Minkowski spacetime. This permits the construction of a regular stress-energy tensor whose vanishing divergence determines a system of non-linear partial differential equations for the velocity and self-fields of accelerated charge. Within this covariant framework a particular perturbation scheme is motivated by an exact class of solutions to this system describing the evolution of a charged fluid under the combined effects of both self and external electromagnetic fields. The scheme yields an asymptotic approximation in terms of inhomogeneous linear equations for the self-consistent Maxwell field, charge current and time-like velocity field of the charged fluid and is defined as an ultra-relativistic configuration. To facilitate comparisons with existing accounts of beam dynamics an appendix translates the tensor formulation of the perturbation scheme into the language involving electric and magnetic fields observed in a laboratory (inertial) frame.
\end{abstract}

\section{Introduction}

The intense international activity involved in probing the structure of matter on all scales, with particle beams and radiation, owes much to recent advances in accelerator science and technology. Developments in the production of high power laser radiation also offer new avenues for accelerator design and new diagnostic tools of relevance to medical science, engineering and the communications industry. A common theme in these developments is the interaction between charged particles and the electromagnetic field in domains where relativistic effects cannot be ignored.

It is remarkable that many of the challenges that must be addressed in order to develop and control devices that accelerate charged particles have their origin in the interaction of particles with their own electromagnetic field. Despite the fact that the classical laws of electromagnetism were essentially formulated over a century and a half ago the subject of electromagnetic interactions with matter remains incomplete. This incompleteness has had concomitant effects on the development of quantum electrodynamics and renormalisation theory. At root, the difficulties reside in the recognition that 
the quantum structure of matter at some scale is beyond observation. Furthermore, the classical description of the electron as a point particle leads to singularities in the Maxwell self-fields that inevitably create ambiguities in its interaction with the Maxwell field. The general consensus is that a useful domain of validity of the Lorentz-Dirac equation [1, 2, 3, describing covariantly the radiation reaction on a point electron, can be accommodated by performing a "reduction of order" that effectively replaces the equation by a perturbative second order system for the particle world line. One must then decide whether higher order terms in this expansion should be maintained given the neglect of terms associated with the regularisation scheme. This approach is behind many of the successful applications of approximate radiation reaction dynamics, despite the somewhat delicate and unsatisfactory nature of the arguments that purport to support this approach.

It appears that the analysis of systems involving radiating matter has progressed by a very successful symbiosis between experimental expediency and a variety of approximation schemes with proven effectiveness in different domains of validity. However, as schemes for accelerating charged particles in these devices become more complex and ambitious in their aims it is apparent that some existing theoretical models are inadequate for a proper understanding of new challenges. Such models are sometimes enshrined in large commercial computer codes that do not survive scientific scrutiny or have documentation that makes contact with established scientific literature difficult. As particle energies increase with the use of higher intensity laser fields this issue becomes critical and more reliable methods for accommodating radiation reaction must be found.

A mathematically coherent formulation of a closed system of partial differential equations describing the relativistic behaviour of charged matter with electromagnetic fields is inevitably non-linear and, in general, exact solutions satisfying causal boundary conditions are intractable. The fact that the effects of (coherent and incoherent) radiation on micrometre-sized charged bunches in a host of newly proposed advanced devices is a significant barrier to development indicates that existing approaches used to model such effects are inadequate and that a new look at the whole problem is timely.

Many of the radiation problems alluded to above can be circumvented by working entirely in the language of fields rather than both particles and fields. This makes it possible to use intrinsic tensor field theory and exploit to the full the power of differential geometry in a relativistic spacetime framework. The geometric field formulation offers a number of powerful computational advantages over existing formulations of beam dynamics. These include the maintenance of relativistic covariance (local Lorentz transformation between local (accelerating) frames are unnecessary), the use of curvilinear coordinates adapted to the geometry of particular problems (thereby facilitating imposition of boundary conditions), exploitation of available symmetries and the formulation of coordinate-independent approximation schemes.

In this article a new approach is offered for analysing the behaviour of distributions of charged particles in a coupled electromagnetic field environment. Guided by a simple model, an approximation scheme designed for charged distributions containing ultra-relativistic particles ${ }^{1}$ is explored. The exact equations of motion are derived from the vanishing divergence of a relativistic stress-energy tensor for matter and radiation. Before presenting the approximation scheme in detail we recall the genesis of the radiation reaction for a point charge, mention its shortcomings and argue that these may be overcome by treating a large collection of charged particles as a continuum.

\footnotetext{
${ }^{1}$ A novel definition of an "ultra-relativistic vector field" relying entirely on the light-cone structure of spacetime is given in Appendix A.1
} 


\subsection{Equations of motion for radiating charges}

In this paper the language of differential geometry is employed since it offers the most succinct mathematical framework for electrodynamics. Thus, all fields will be regarded as sections of tensor bundles over appropriate domains $\mathcal{M}$ of Minkowski spacetime endowed with a fixed metric $g$ and a torsion-free metric compatible connection $\nabla$. Sections of the tangent bundle over $\mathcal{M}$ will be denoted $\Gamma T \mathcal{M}$ while sections of the bundle of exterior $p$-forms will be denoted $\Gamma \Lambda^{p} \mathcal{M}$. For any vector field $X$ denote by $\widetilde{X}$ the associated 1 -form defined by $\widetilde{X}=g(X,-)$. The operator $d$ will denote the exterior derivative and $i_{X}$ the contraction operator with respect to $X$. To facilitate comparisons with existing accounts of beam dynamics Appendix A.2 translates the tensor formulation of the perturbation scheme into the 3-vector language involving electric and magnetic fields observed in a laboratory (inertial) frame.

Due to its high symmetry, Minkowski spacetime admits a class of global charts that play a fundamental role in the following. A generic system of coordinates in one of these charts will be denoted $\left\{y^{\mu}\right\} \in \mathbb{R}^{4}$ where $\mu$ ranges from 0 to 3 . In these coordinates the metric tensor takes the form

$$
g=-d y^{0} \otimes d y^{0}+d y^{1} \otimes d y^{1}+d y^{2} \otimes d y^{2}+d y^{3} \otimes d y^{3} .
$$

With $y^{0}>0$ the field $\frac{\partial}{\partial y^{0}}$ is defined to be future-pointing. When convenient $y^{0}=c t, y^{1}=x, y^{2}=$ $y, y^{3}=z$ is written and the vacuum speed of light $c$ is set to 1 . The significance of this class of coordinates is that it offers a basis of symmetry generators $\left\{K_{\mu}=\frac{\partial}{\partial y^{\mu}}\right\}$ :

$$
\mathcal{L}_{K_{\mu}} g=0
$$

where $\mathcal{L}_{X}$ is the Lie derivative with respect to $X$. Such Killing vectors will be used to define energymomentum densities and power-momentum fluxes associated with different field configurations on spacetime.

If $\mathcal{U}$ is a domain of spacetime with boundary

$$
\partial \mathcal{U}=\Sigma_{1}+\Sigma_{2}+\Pi
$$

for space-like hypersurfaces $\Sigma_{1}, \Sigma_{2}$ and $\mathcal{J}$ a closed regular 3 -form on $\mathcal{U}$ (i.e. $d \mathcal{J}=0$ ) then

$$
\int_{\partial \mathcal{U}} \mathcal{J}=\int_{\mathcal{U}} d \mathcal{J}=0 .
$$

Thus

$$
\int_{\Sigma_{1}} \mathcal{J}=\int_{-\Sigma_{2}} \mathcal{J}-\int_{\Pi} \mathcal{J}
$$

Such closed 3-forms imply conservation laws.

In a vacuum the electric and magnetic fields are encoded into the 2 -form $\mathcal{F}$ and the electric charge density and current are described by a source current 3-form $j$. The Maxwell field system on spacetime is

$$
d \mathcal{F}=0
$$

and

$$
d \star \mathcal{G}=-j
$$

where $\star$ is the Hodge map associated with the spacetime metric tensor $g$ and $\mathcal{G}=\epsilon_{0} \mathcal{F}$ where $\epsilon_{0}$ is the permittivity of the vacuum. 
For any vector field $W$ on spacetime and any Maxwell solution $\mathcal{F}$ define the "electromagnetic drive" 3-form

$$
\tau_{W}^{(\mathrm{EM})}=\frac{1}{2}\left\{i_{W} \mathcal{F} \wedge \star \mathcal{F}-i_{W} \star \mathcal{F} \wedge \mathcal{F}\right\} .
$$

If $W$ is a Killing vector field then $\tau_{W}^{(\mathrm{EM})}$ is called a "Killing current" and

$$
d \tau_{W}^{(\mathrm{EM})}=\frac{1}{\epsilon_{0}} i_{W} \mathcal{F} \wedge j
$$

follows from (6), (7) and (8). For each Killing vector field these equations establish a "local conservation equation" $\left(d \tau_{W}=0\right)$ in a source free region $(j=0)$.

If $U$ is any time-like vector field (with $g(U, U)=-c^{2}$ ) one may write uniquely

$$
\mathcal{F}=\tilde{e} \wedge \tilde{U}+\star(\tilde{b} \wedge \tilde{U})
$$

where $g(e, U)=g(b, U)=0$ and

$$
\frac{1}{c^{2}} \tau_{U}^{(\mathrm{EM})}=-\tilde{e} \wedge \tilde{b} \wedge \tilde{U}-\frac{1}{2}\{g(e, e)+g(b, b)\} i_{U}(\star 1) .
$$

Poynting $^{2}$ related the form $\tilde{e} \wedge \tilde{b}$, in a source free region, to the local field energy transmitted normally across unit area per second (spatial field energy current or field power) and $\frac{1}{2}\{g(e, e)+g(b, b)\}$ to the local electromagnetic field energy density.

More precisely $\frac{1}{c} \int_{\Sigma} \tau_{U}^{(\mathrm{EM})}$ is the field energy associated with the space-like 3 -chain $\Sigma$ and $\frac{1}{c^{2}} \int_{S} i_{U} \tau_{U}^{(\mathrm{EM})}$ is the power flux across an oriented space-like 2-chain $S$.

If $X$ is a space-like Killing vector generating space-like translations along open integral curves then with the split:

$$
\tau_{X}^{(\mathrm{EM})}=\mu_{X} \wedge \tilde{U}+\mathcal{G}_{X}
$$

where $i_{U} \mu_{X}=0$ and $i_{U} \mathcal{G}_{X}=0$, the Maxwell stress 2-form $\mu_{X}$ may be used to identify mechanical forces produced by a flow of field momentum with density 3-form $\mathcal{G}_{X}$. In any local frame $\left\{X_{a} \in\right.$ $\Gamma T \mathcal{M}\}$ with dual coframe $\left\{e^{b} \in \Gamma \Lambda^{1} \mathcal{M}\right\}$ the 16 functions $T_{a b}^{(\mathrm{EM})}=i_{X_{b}} \star \tau_{X_{a}}^{(\mathrm{EM})}$, where the frame indices $a, b=0,1,2,3$, may be used to construct the second-rank stress-energy tensor

$$
T^{(\mathrm{EM})}=T_{a b}^{(\mathrm{EM})} e^{a} \otimes e^{b} .
$$

A standard application of these general notions is the derivation of the equation of motion of a charged fluid in an external electromagnetic field from the total stress-energy of the fluid and electromagnetic field. A thermodynamically inert (cold) fluid can be modelled with the stress-energy tensor

$$
T^{(\mathrm{f})}=\frac{m_{0}}{c \epsilon_{0}} \mathcal{N} \widetilde{V} \otimes \widetilde{V}
$$

where $\mathcal{N}$ is a scalar number density field, $m_{0}$ some constant with the dimensions of mass, $V$ the unit time-like 4-velocity field of the fluid and $g(V, V)=-1$. Such a stress-energy tensor gives rise to a set of Killing currents

$$
\tau_{K_{\mu}}^{(\mathfrak{f})}=\frac{m_{0}}{c \epsilon_{0}} g\left(V, K_{\mu}\right) \star(\mathcal{N} \widetilde{V})
$$

\footnotetext{
${ }^{2}$ In a pre-relativistic context.
} 
which are added to $\tau_{K_{\mu}}^{(\mathrm{EM})}$ to yield the total set of Killing currents for the interacting system. If one assumes that the electric current 3 -form is $j=q_{0} \mathcal{N} \star \widetilde{V}$ for some electric charge constant $q_{0}$ and that $\mathcal{N}$ is regular then the conservation laws

$$
\begin{gathered}
d j=0 \\
d\left(\tau_{K_{\mu}}^{(\mathrm{EM})}+\tau_{K_{\mu}}^{(\mathrm{f})}\right)=0
\end{gathered}
$$

yield the field equation of motion

$$
\nabla_{V} \widetilde{V}=\frac{q_{0}}{m_{0} c^{2}} i_{V} \mathcal{F}
$$

This equation must be solved consistently with the Maxwell equations (6), (77) to determine $V, \mathcal{N}$ and $\mathcal{F}$ for prescribed initial and boundary conditions.

If the source of $\mathcal{F}$ contains a point charge in an external field this approach must take into account that part of the electromagnetic field becomes singular on the world-line of the particle so the domain $\mathcal{U}$ above must exclude this line. Furthermore, one needs to postulate a stress-energy tensor for a stable point particle. Without further information application of Stokes' theorem then leaves an unknown contribution to the conservation law. However, experiment indicates that accelerating electrons (thought to be point particles) experience a reaction to their emitted radiation and that the power radiated is in good agreement with the covariant Larmor formula [4]. A number of methods have been devised in an attempt to accommodate this observed radiation reaction by adopting a regularisation procedure that permits the use of Stokes' theorem in a spacetime region that includes the particle world-line. Inevitably this requires some assumption of how the singular Coulombic stresses are compensated by stresses that are not of electromagnetic origin. Early approaches invoked delicate limiting processes and the use of both advanced as well as retarded solutions to Maxwell's equations [5, 3]. Dirac [5] offered one of the simplest covariant regularisation schemes and the resulting equation of motion is known as the Lorentz-Dirac equation. The use of both advanced and retarded solutions is however unnecessary [6, 7].

To highlight the assumptions in the single particle approach to radiation reaction an outline is given of how the Lorentz-Dirac equation can be derived using a coordinate system adapted to the light-cone structure of spacetime and the time-like world-line of a particle on a parametrised history given in the above chart as $y^{\mu}=\xi^{\mu}(u)$. For each value of $u$ the unit tangent to the world-line is the 4-velocity

$$
V=V^{\mu}(u) \frac{\partial}{\partial y^{\mu}}
$$

where $V^{\mu}(u)=\frac{d \xi^{\mu}(u)}{d u}$.

Assign to any event in spacetime not on the particle world-line, whose backward light-cone intersects this world-line at the event with parameter $u$, the set of coordinates $\{u, r, \theta, \phi\}$ with $0 \leq r \leq \infty, 0<\theta \leq \pi, 0<\phi \leq 2 \pi$ using the transformation

$$
y^{\mu}=\xi^{\mu}(u)+\frac{r}{\mathcal{P}(u, \theta, \phi)} L^{\mu}(\theta, \phi),
$$

where for any $y^{0}>0$ :

$$
L=\frac{\partial}{\partial y^{0}}+\sum_{k=1}^{3} n^{k}(\theta, \phi) \frac{\partial}{\partial y^{k}}=L^{\mu} \frac{\partial}{\partial y^{\mu}}
$$




$$
\begin{gathered}
\sum_{k=1}^{3}\left(n^{k}(\theta, \phi)\right)^{2}=1 \\
\mathcal{P}=-g(V, L), \quad g(V, V)=-1, \quad g(L, L)=0 .
\end{gathered}
$$

In these coordinates the particle world-line is the curve where ${ }^{3} r=0,(\theta, \phi)$ parametrise the unit 2-sphere $S^{2}$ where $\left\{n^{k}(\theta, \phi)\right\}$ are direction cosines and the metric tensor can be written:

$$
g=-e^{0} \otimes e^{0}+e^{1} \otimes e^{1}+e^{2} \otimes e^{2}+e^{3} \otimes e^{3}
$$

where

$$
\begin{gathered}
e^{0}=\mathcal{B} d u-\frac{1}{\mathcal{B}} d r \\
e^{1}=\frac{d r}{\mathcal{B}} \\
e^{2}=\frac{r}{\mathcal{P}} d \theta \\
e^{3}=\frac{r}{\mathcal{P}} \sin \theta d \phi \\
\mathcal{B}=\sqrt{1+2 r \frac{\dot{\mathcal{P}}}{\mathcal{P}}}
\end{gathered}
$$

Here, and in the following, denote $\partial_{u} f$ by $\dot{f}$ for any scalar field $f$ and let $\dot{\mathcal{T}}=\mathcal{L}_{\frac{\partial}{\partial u}} \mathcal{T}$ for any tensor field $\mathcal{T}$.

Suppose the total system (electromagnetic field and charge) has a stress-energy tensor giving rise to a regular Killing current $\tau_{K}$ such that

$$
d \tau_{K}=0
$$

in a domain $\mathcal{U}$ bounded by the light-like 3-chains $\Sigma\left(u=u_{0}+\Delta u\right), \Sigma\left(u=u_{0}\right)$ and the time-like 3-chain $\Sigma\left(r=r_{0}\right)$ for arbitrary positive constants $u_{0}, \Delta u, r_{0}$. Then

$$
\int_{\Sigma\left(u=u_{0}+\Delta u\right)} \tau_{K}-\int_{\Sigma\left(u=u_{0}\right)} \tau_{K}+\int_{\Sigma\left(r=r_{0}\right)} \tau_{K}=0 .
$$

In the limit $\Delta u \rightarrow 0$ this may be written

$$
\dot{P}_{K}^{(\mathrm{B})}\left(u_{0}, r_{0}\right) d u+\dot{P}_{K}^{(\mathrm{C})}\left(u_{0}, r_{0}\right) d u=0
$$

where

$$
\begin{aligned}
& \dot{P}_{K}^{(\mathrm{B})}\left(u_{0}, r_{0}\right)=\int_{0}^{r_{0}} \int_{S^{2}} \dot{\tau}_{K}\left(u_{0}, r, \theta, \phi\right) \\
& \dot{P}_{K}^{(\mathrm{C})}\left(u_{0}, r_{0}\right)=\int_{S^{2}} i_{\partial_{u}} \tau_{K}\left(u_{0}, r_{0}, \theta, \phi\right)
\end{aligned}
$$

\footnotetext{
${ }^{3}$ For $\mathcal{P}>0$ with $V$ time-like and $\frac{\partial}{\partial y^{0}}$ future-pointing the set $r \geq 0$ lies on the forward light-cone of the event $\xi^{\mu}(u)$.
} 
With $K \in\left\{\frac{\partial}{\partial y^{\mu}}\right\}$ and $\nabla \frac{\partial}{\partial y^{\mu}}=0$ one has a similar balance of rates for each translational Killing vector in the basis. In terms of the "body" rate

$$
\dot{P}^{(\mathrm{B})} \equiv \dot{P}_{\frac{\partial}{\partial y^{\mu}}}^{(\mathrm{B})} d y^{\mu}
$$

and the "contact" rate

$$
\dot{P}^{(\mathrm{C})} \equiv \dot{P}_{\frac{\partial}{\partial y^{\mu}}}^{(\mathrm{C})} d y^{\mu}
$$

there is a balance of 1 -forms:

$$
\dot{P}^{(\mathrm{B})}\left(u_{0}, r_{0}\right)+\dot{P}^{(\mathrm{C})}\left(u_{0}, r_{0}\right)=0 .
$$

Next assume that the contact rate includes a part from the Killing currents $\tau_{K}^{(\mathrm{EM})}$, i.e.

$$
\dot{P}^{(\mathrm{C})}=\dot{P}_{\mathrm{MECH}}^{(\mathrm{C})}+\dot{P}_{\mathrm{EM}}^{(\mathrm{C})}
$$

and furthermore that this part can be calculated from the retarded Liénard-Wiechert solution $\mathcal{F}$ to Maxwell's equations for an arbitrarily moving point charge $q_{0}$ in no external electromagnetic field. In these coordinates the solution is $\mathcal{F}=d A$ where the 1 -form

$$
A=\frac{q_{0}}{4 \pi \epsilon_{0}} \frac{\widetilde{V}(u)}{r}
$$

Integration over the 2-chain $S^{2}$ with $r=r_{0}$ yields for (33) :

$$
\dot{P}_{\mathrm{EM}}^{(\mathrm{C})}=\left.\frac{q_{0}^{2}}{4 \pi \epsilon_{0}^{2}}\left\{\frac{2}{3} \widetilde{\mathcal{A}}(\mathcal{A}) \widetilde{V}+\frac{\widetilde{\mathcal{A}}}{2 r_{0}}\right\}\right|_{u=u_{0}}
$$

where $\mathcal{A}=\dot{V}^{\mu}(u) \frac{\partial}{\partial y^{\mu}}$ is the acceleration field. Clearly this 1-form is singular on the world-line where $r_{0}=0$. The first term however correctly accounts for the observed Larmor radiation rate of energymomentum from an accelerating charge and is independent of $r_{0}$. To proceed one must cancel the singular rate in $\dot{P}_{\mathrm{EM}}^{(\mathrm{C})}$ from singular terms in the remaining rates in such a way that in the limit $r_{0} \rightarrow 0$ the resulting system of ordinary differential equations for arbitrary $u_{0}$ makes both mathematical and physical sense. One approach is to suppose that the remaining rates are determined in terms of scalar fields $\alpha(u, r), \beta(u, r)$ and the vectors $V, \mathcal{A}$ such that

$$
P^{(\mathrm{B})}\left(u, r_{0}\right)+P_{\mathrm{MECH}}^{(\mathrm{C})}\left(u, r_{0}\right)=\alpha\left(u, r_{0}\right) \widetilde{V}\left(u, r_{0}\right)+\beta\left(u, r_{0}\right) \widetilde{\mathcal{A}}\left(u, r_{0}\right) .
$$

Inserting this in (36) and applying the projection operator $\Pi_{V}=1+\widetilde{V} \wedge i_{V}$ one finds

$$
\beta=-\frac{2}{3} \frac{q_{0}^{2}}{4 \pi \epsilon_{0}^{2}}+\frac{\dot{\alpha}}{\widetilde{\mathcal{A}}(\mathcal{A})}
$$

With this value (36) yields

$$
\widetilde{\mathcal{A}}\left[\alpha-\frac{d}{d u}\left(\frac{\dot{\alpha}}{\widetilde{\mathcal{A}}(\mathcal{A})}\right)+\frac{q_{0}^{2}}{4 \pi \epsilon_{0}^{2}} \frac{1}{2 r_{0}}\right]=-\widetilde{V}\left[\dot{\alpha}+\frac{2}{3} \frac{q_{0}^{2}}{4 \pi \epsilon_{0}^{2}} \widetilde{\mathcal{A}}(\mathcal{A})\right]+\left[\frac{2}{3} \frac{q_{0}^{2}}{4 \pi \epsilon_{0}^{2}}+\frac{\dot{\alpha}}{\widetilde{\mathcal{A}}(\mathcal{A})}\right] \widetilde{\mathcal{A}}
$$

where $\dot{\mathcal{A}}=\ddot{V}^{\mu}(u) \frac{\partial}{\partial y^{\mu}}$. One way to simplify this and cancel the exposed electromagnetic singularity is to further assume that $\alpha\left(u, r_{0}\right)=\frac{m_{0} c^{2}}{\epsilon_{0}}-\frac{q_{0}^{2}}{4 \pi \epsilon_{0}^{2}} \frac{1}{2 r_{0}}$ for some constant $m_{0}$ so that in the limit $r_{0} \rightarrow 0$ one has:

$$
m_{0} c^{2} \widetilde{\mathcal{A}}=\frac{2}{3} \frac{q_{0}^{2}}{4 \pi \epsilon_{0}} \Pi_{V} \widetilde{\mathcal{A}}
$$


or

$$
m_{0} c^{2} \widetilde{\mathcal{A}}=-\frac{2}{3} \frac{q_{0}^{2}}{4 \pi \epsilon_{0}} i_{V}(\widetilde{V} \wedge \widetilde{\mathcal{A}})
$$

This is a manifestly covariant equation of motion even though the velocity and acceleration are specified in a basis of Killing vector fields. Since it is tensorial it is independent of basis and in terms of covariant differentiation along the world-line it may be written

$$
m_{0} c^{2} \widetilde{\mathcal{A}}=-\frac{2}{3} \frac{q_{0}^{2}}{4 \pi \epsilon_{0}} i_{V}\left(\widetilde{V} \wedge \nabla_{V} \widetilde{\mathcal{A}}\right)
$$

This is a system of third order differential equations for the worldline $\xi^{\mu}(u)$. Regarded as an initial value problem it requires unfamiliar initial data $\left(\xi^{\mu}(0), \dot{\xi}^{\mu}(0), \ddot{\xi}^{\mu}(0)\right)$ and solutions exist corresponding to self acceleration which must be regarded as unphysical.

In the presence of an external Maxwell field $\mathcal{F}_{\text {ext }}$ one must confine the motion of the particle to a domain $\mathcal{U}$ that excludes the sources of $\mathcal{F}_{\text {ext }}$. In this situation one expects that the equation of motion will acquire a contribution from the Lorentz force $q_{0} i_{V} \mathcal{F}_{\text {ext }}$ :

$$
m_{0} c^{2} \widetilde{\mathcal{A}}=q_{0} i_{V} \mathcal{F}_{\text {ext }}+\frac{2}{3} \frac{q_{0}^{2}}{4 \pi \epsilon_{0}} \Pi_{V} \widetilde{\mathcal{A}}
$$

so that $m_{0}$ is identified with the rest mass of the point particle.

Although solutions to this system that self-accelerate can be eliminated by demanding contrived data at different points along the world-line there remain solutions that pre-accelerate in situations where the external field is piecewise defined in spacetime 3. Although the duration of preacceleration is probably classically unobservable for most electrons this general feature suggests that not all of the assumptions above are acceptable.

One resolution of these difficulties [1] is to assume that the right hand side of (46) should be expanded as a series in $q_{0}$ with leading term for $\tilde{\mathcal{A}}$ given by $\frac{q_{0}}{m c^{2}} i_{V} \mathcal{F}_{\text {ext }}$. Then the above assumptions are held to be accurate only to some order in $q_{0}$ and (46) is understood as:

$$
\widetilde{\mathcal{A}}=\frac{q_{0}}{m_{0} c^{2}} i_{V} \mathcal{F}_{\text {ext }}-\frac{2}{3 m_{0} c^{2}} \frac{q_{0}^{2}}{4 \pi \epsilon_{0}} i_{V}\left(\widetilde{V} \wedge \nabla_{V} \widetilde{\mathcal{A}}_{\mathrm{ext}}\right)+\ldots
$$

where $\widetilde{\mathcal{A}}_{\text {ext }}=\frac{q_{0}}{m_{0} c^{2}} i_{V} \mathcal{F}_{\text {ext }}$. The system is now manifestly a second order system of evolution equations. Although this offers a workable scheme it is unclear what its limitations are in different types of external field. Furthermore in situations where one has to contemplate the radiation from a large number of accelerating high-energy particles in close proximity the neglect of higher order terms in the expansion may be suspect.

Given the complexities and reservations associated with (47) compared with the model leading to (17) the latter is adopted in this article as a description of a collection of potentially radiating particles in the presence of an external electromagnetic field. Thus, our fundamental set of equations is

$$
\begin{aligned}
& d \mathcal{F}=0, \\
& d \star \mathcal{F}=-\frac{q_{0}}{\epsilon_{0}} \mathcal{N} \star \widetilde{V}, \\
& \nabla_{V} \widetilde{V}=\frac{q_{0}}{m_{0} c^{2}} i_{V} \mathcal{F}, \\
& V \cdot V=-1
\end{aligned}
$$


where $X \cdot Y \equiv g(X, Y)$ for all vector fields $X, Y \in \Gamma T \mathcal{M}$.

To simplify the subsequent analysis the system (48.51) is recast using the 2 -form $F=\frac{q_{0}}{m_{0} c^{2}} \mathcal{F}$ and the scalar field $\rho=\frac{q_{0}^{2}}{\epsilon_{0} m_{0} c^{2}} \mathcal{N}$ where $q_{0} \mathcal{N}$ is the proper charge density since $\mathcal{N}$ is the proper number density. This yields the system of field equations

$$
\begin{aligned}
& d F=0, \\
& d \star F=-\rho \star \widetilde{V}, \\
& \nabla_{V} \widetilde{V}=i_{V} F, \\
& V \cdot V=-1
\end{aligned}
$$

for the triple $(V, \rho, F)$. Equation (53) leads immediately to the integrability condition (conservation of electric charge)

$$
d \star(\rho \widetilde{V})=0 .
$$

The scalar field $\rho$ will be referred to as the reduced proper charge density. Since $\mathcal{N}$ is a number density, $\mathcal{N} \geq 0$ and so $\rho \geq 0$ for $q_{0}>0$ and $q_{0}<0$.

\section{Symmetric solutions to the coupled field system}

Although the field system (52,55) appears considerably less complicated than a large number of ordinary differential equations for a collection of accelerating charges, it is non-linear in $(V, \rho, F)$ and so obtaining exact solutions is difficult except in certain simple circumstances. One method of obtaining solutions in more general situations is to employ a perturbation scheme based on properties of a particular class of exact solutions.

In this section a family of exact dynamical solutions with symmetries generated by $\frac{\partial}{\partial y^{1}}$ and $\frac{\partial}{\partial y^{2}}$ will be described. Such highly symmetric solutions can be interpreted as accelerating "walls of charge" moving in vacuo under the influence of their self-fields (their "space-charge" fields) and an externally applied field. The coordinate $y^{0}$ will be identified with time $t$ in an inertial laboratory frame (the speed of light $c=1$ ) and $x \equiv y^{1}, y \equiv y^{2}$, and $z \equiv y^{3}$. The solutions will be independent of $y^{1}$ and $y^{2}$ i.e.

$$
\begin{aligned}
& \mathcal{L}_{\frac{\partial}{\partial y^{1}}} F=\mathcal{L}_{\frac{\partial}{\partial y^{2}}} F=0, \\
& \mathcal{L}_{\frac{\partial}{\partial y^{1}}} V=\mathcal{L}_{\frac{\partial}{\partial y^{2}}} V=0 .
\end{aligned}
$$

Thus one may reduce the problem to a field theory on a 2-dimensional Lorentzian spacetime with global coordinates $y^{0}, y^{3}$. The field system (52,55) is solved exactly using a co-moving coordinate system $(\tau, \sigma)$ adapted to the charged continuum. However, expressing the solutions in terms of laboratory coordinates $(t, z)$ requires the inverse of the mapping $(\tau, \sigma) \rightarrow(t, z)$, which is generally difficult to obtain in closed form. To progress, a running parameter $\varepsilon>0$ is introduced into the mapping $(\tau, \sigma) \rightarrow(t, z)$ and a perturbation scheme in $\varepsilon$ is established. This approach facilitates an order-by-order construction of the inverse of the mapping $(\tau, \sigma) \rightarrow(t, z)$ and thereby leads to 1-parameter families $\left(V^{\varepsilon}, \rho^{\varepsilon}, F^{\varepsilon}\right)$ of solutions in $\varepsilon$. It will be shown that

$$
F^{\varepsilon}=\sum_{n=-1}^{\infty} \varepsilon^{n} F_{n}, \quad V^{\varepsilon}=\sum_{n=-1}^{\infty} \varepsilon^{n} V_{n}, \quad \rho^{\varepsilon}=\sum_{n=1}^{\infty} \varepsilon^{n} \rho_{n}
$$


over some range of $\varepsilon$ where the coefficients $F_{n}, V_{n}$ and $\rho_{n}$ are 2-forms, vector fields and scalar fields respectively.

Exact solutions to the system of equations (52,55) are obtained using the ansätz

$$
\begin{aligned}
& F=\mathcal{E}(t, z) d t \wedge d z \\
& V=\frac{1}{\sqrt{1-\mu^{2}(t, z)}}\left(\partial_{t}+\mu(t, z) \partial_{z}\right)
\end{aligned}
$$

where $\mu$ is the magnitude of the Newtonian velocity field of the charged continuum measured by the inertial (laboratory) observer $\partial_{t}$. Equations (52) and (55) are manifestly satisfied while (53) and (54) lead to

$$
\begin{aligned}
& d \mathcal{E}=\rho \# \widetilde{V}, \\
& \nabla_{V} \widetilde{V}=\mathcal{E} \# \widetilde{V}
\end{aligned}
$$

where \# is the Hodge map associated with the volume 2 -form $\# 1 \equiv d t \wedge d z$. Since $i_{V} \# \alpha=\#(\alpha \wedge \widetilde{V})$ for all forms $\alpha$ independent of $d x$ and $d y$, the action of $i_{V}$ on (57) yields

$$
V \mathcal{E}=0
$$

i.e. $\mathcal{E}$ is constant along the integral curves of $V$. Let $C_{\sigma}$ be a 1-parameter family of proper-timeparametrised integral curves of $V$ where each value of $\sigma$ corresponds to an integral curve of $V$ :

$$
\begin{aligned}
C_{\sigma}: \mathbb{R} & \rightarrow \mathcal{M}, \\
\tau & \rightarrow(t=\hat{t}(\tau, \sigma), z=\hat{z}(\tau, \sigma))
\end{aligned}
$$

and

$$
V=C_{\sigma *} \partial_{\tau}=\frac{\partial \hat{t}}{\partial \tau} \partial_{t}+\frac{\partial \hat{z}}{\partial \tau} \partial_{z}
$$

where $C_{\sigma *}$ is the push-forward (tangent) map associated with $C_{\sigma}$. Equation (59) is

$$
\begin{aligned}
\left(C_{\sigma *} \partial_{\tau}\right) \mathcal{E} & =\partial_{\tau}\left(C_{\sigma}^{*} \mathcal{E}\right) \\
& =0
\end{aligned}
$$

where $C_{\sigma}^{*}$ is the pull-back map associated with $C_{\sigma}$. Hence $C_{\sigma}^{*} \mathcal{E}$ depends only on $\sigma$ and can be expressed using the function $\zeta: \mathbb{R} \rightarrow \mathbb{R}$ where

$$
\begin{aligned}
\zeta(\sigma) & \equiv C_{\sigma}^{*} \mathcal{E} \\
& =\mathcal{E}(\hat{t}(\tau, \sigma), \hat{z}(\tau, \sigma)) .
\end{aligned}
$$

Using $\nabla \partial_{t}=\nabla \partial_{z}=0$ the $(t, z)$ components of (158) are

$$
\begin{aligned}
& \frac{\partial^{2} \hat{t}}{\partial \tau^{2}}(\tau, \sigma)=\zeta(\sigma) \hat{z}(\tau, \sigma), \\
& \frac{\partial^{2} \hat{z}}{\partial \tau^{2}}(\tau, \sigma)=\zeta(\sigma) \hat{t}(\tau, \sigma)
\end{aligned}
$$


whose particular solution satisfying the initial conditions

$$
\begin{aligned}
& \hat{t}(0, \sigma)=0, \hat{z}(0, \sigma)=\sigma, \\
& \frac{\partial \hat{t}}{\partial \tau}(0, \sigma)=1, \frac{\partial \hat{z}}{\partial \tau}(0, \sigma)=0,
\end{aligned}
$$

at $\tau=0$ is

$$
\begin{aligned}
& t=\hat{t}(\tau, \sigma)=\frac{1}{\zeta(\sigma)} \sinh (\zeta(\sigma) \tau) \\
& z=\hat{z}(\tau, \sigma)=\frac{1}{\zeta(\sigma)}[\cosh (\zeta(\sigma) \tau)-1]+\sigma
\end{aligned}
$$

where the function $\zeta: \mathbb{R} \rightarrow \mathbb{R}$ must be supplied as data (i.e. $\zeta$ is an ingredient in the initial conditions). Note that the charged continuum is taken to be initially at rest in the laboratory, i.e.

$$
\left.V\right|_{\tau=0}=\left.\left(C_{\sigma *} \partial_{\tau}\right)\right|_{\tau=0}=\partial_{t}
$$

More general initial conditions could have been used, but the above scenario is sufficient.

After $\zeta$ has been specified, expressions for $V$ and $F$ in the $(t, z)$ coordinate system are obtained by inverting (62) and (63) to give $(\tau, \sigma)$ in terms of $(t, z)$ :

$$
\begin{aligned}
\tau & =\hat{\tau}(t, z), \\
\sigma & =\hat{\sigma}(t, z)
\end{aligned}
$$

and using (60) it follows that

$$
\mathcal{E}(t, z)=\zeta(\hat{\sigma}(t, z))
$$

An initial condition on $\mathcal{E}$ is expressed using $\zeta$. Equation (62) is used to show that the local hypersurfaces $t=0$ and $\tau=0$ are equal and so (63) yields

$$
\hat{\sigma}(0, z)=z
$$

Thus, (60) leads to

$$
\begin{aligned}
\mathcal{E}(0, z) & =\zeta(\hat{\sigma}(0, z)) \\
& =\zeta(z)
\end{aligned}
$$

on the space-like hypersurface $t=0$.

Not all choices for $\zeta$ are admissible because, as noted in the derivation of (52,55), the reduced proper charge density $\rho$ is positive:

$$
\rho=-i_{V}(\# d \mathcal{E}) \geq 0
$$

using (57) and (55). At $t=\tau=0$

$$
\begin{aligned}
\rho(0, z) & =-i_{\partial_{t}} \#\left(\partial_{t} \mathcal{E}(0, z) d t+\partial_{z} \mathcal{E}(0, z) d z\right) \\
& =\partial_{z} \mathcal{E}(0, z) \\
& =\zeta^{\prime}(z)
\end{aligned}
$$


where $\# d t=-d z$ and $\# d z=-d t$ have been used and $\zeta^{\prime}(z) \equiv \frac{d \zeta}{d z}(z)$. Hence, employing (64)

$$
\zeta^{\prime}(z) \geq 0
$$

i.e. $\zeta$ is a monotonically increasing function.

The coordinate chart $(\tau, \sigma)$ has important physical significance because it labels the streamlines of the charged continuum (the integral curves of $V$ ). Physically, the coordinate transformation $(\tau, \sigma) \rightarrow(t, z)$ is expected to be valid over the entire $(\tau, \sigma)$ plane. This will now be shown.

The determinant $\Delta$ of the Jacobian matrix of the coordinate transformation $(\tau, \sigma) \rightarrow(t, z)$ is

$$
\begin{aligned}
\Delta & \equiv \operatorname{det}\left[\begin{array}{ll}
\frac{\partial \hat{t}}{\partial \tau} & \frac{\partial \hat{z}}{\partial \tau} \\
\frac{\partial \hat{t}}{\partial \sigma} & \frac{\partial \hat{z}}{\partial \sigma}
\end{array}\right] \\
& =-\frac{\zeta^{\prime}(\sigma)}{\zeta(\sigma)^{2}}+\cosh (\zeta(\sigma) \tau)\left(1+\frac{\zeta^{\prime}(\sigma)}{\zeta(\sigma)^{2}}\right)
\end{aligned}
$$

Since $\zeta^{\prime}(\sigma) \geq 0$ and $\cosh (\zeta(\sigma) \tau) \geq 1$ it follows that

$$
\Delta \geq 1
$$

Monotonicity of $\zeta$ implies that $\zeta$ must vanish somewhere. Let $\sigma_{0} \in \mathbb{R}$ be the point where $\zeta\left(\sigma_{0}\right)=0$ and note

$$
\lim _{\sigma \rightarrow \sigma_{0}} \Delta=1+\frac{1}{2} \zeta^{\prime}\left(\sigma_{0}\right) \tau^{2}
$$

using (66). Therefore, $\Delta$ is finite for all $(\tau, \sigma) \in \mathbb{R}^{2}$ and the coordinate transformation $(\tau, \sigma) \rightarrow(t, z)$ is valid over the entire $(\tau, \sigma)$ plane.

The implicit equation

$$
\sigma=z-\frac{1}{\zeta(\sigma)}\left(\sqrt{1+[\zeta(\sigma)]^{2} t^{2}}-1\right)
$$

for $\sigma=\hat{\sigma}(t, z)$ arises by combining (62) with (63).

The initial electric field $\mathcal{E}(0, z)=\zeta(z)=\zeta_{\text {ext }}+\zeta_{\text {self }}(z)$ where $\zeta_{\text {ext }}$ models an externally applied field and $\zeta_{\text {self }}$ is the self-field of the charged continuum. The field $\zeta_{\text {ext }}$ is constant because it satisfies the vacuum Maxwell equation

$$
d \zeta_{\text {ext }}=0
$$

inside the charged continuum (consider equation (157) with $\rho=0$ ). The self-field $\zeta_{\text {self }}$ is to be defined in terms of the initial reduced proper charge density $\rho(0, z)$ :

$$
\zeta_{\text {self }}(z) \equiv \frac{1}{2}\left[\int_{-\infty}^{z} \rho(0, s) d s-\int_{z}^{\infty} \rho(0, s) d s\right]
$$

and satisfies the asymptotic conditions

$$
\begin{aligned}
& \lim _{z \rightarrow \infty} \zeta_{\text {self }}(z)=\frac{1}{2} Q, \\
& \lim _{z \rightarrow-\infty} \zeta_{\text {self }}(z)=-\frac{1}{2} Q
\end{aligned}
$$

where $Q=\int_{-\infty}^{\infty} \rho(0, z) d z$ is the total charge per unit area of the continuum. 


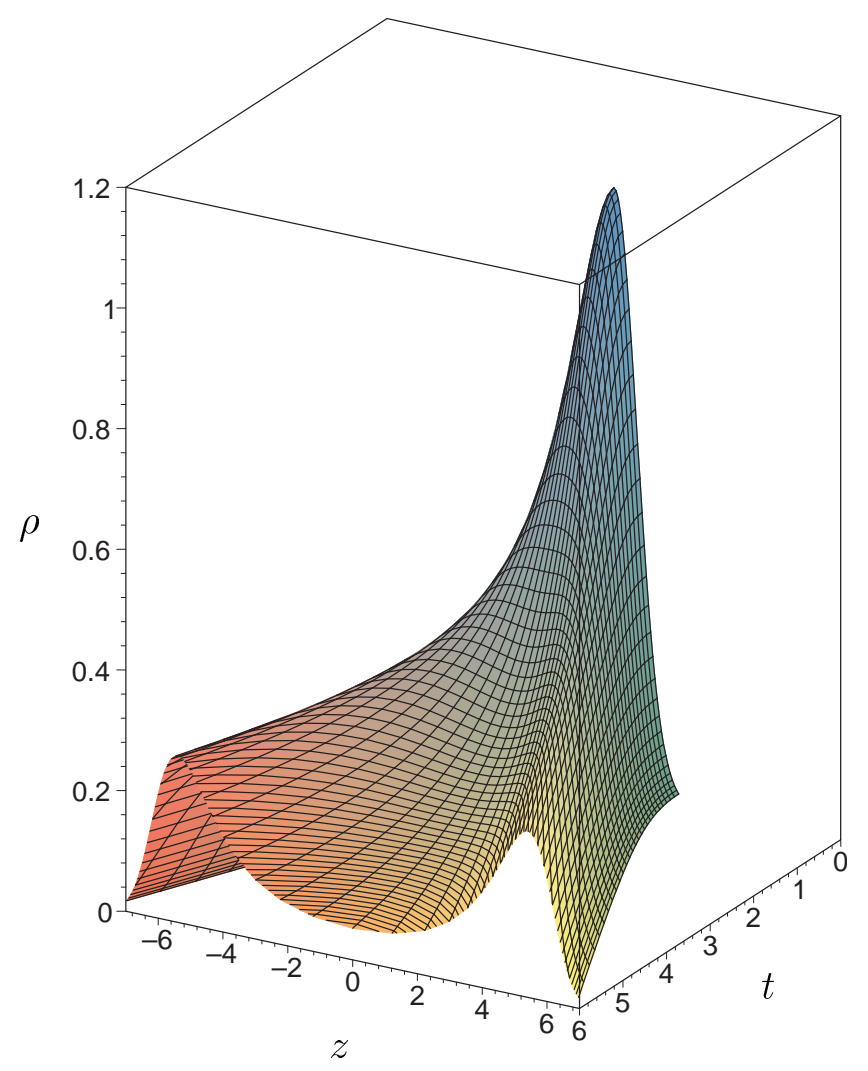

Figure 1: The time history of the initial charge distribution (69) with zero applied external field.

Illustrations of the above results are shown in figures 10. In figures 1 and 2 the external field is set to zero so the charge distribution $\rho$ evolves entirely under its own self-field. In figures 3 and 4 the external field is set to a finite value. Figures 2 and 4 show the streamlines of $V$ in spacetime for each case. The initial charge distribution is taken as the Gaussian

$$
\rho(0, z)=\exp \left(-z^{2}\right)
$$

and, using (68), the associated electric self-field is

$$
\zeta_{\text {self }}(z)=\frac{\sqrt{\pi}}{2} \operatorname{erf}(z)
$$

The external field $\zeta_{\text {ext }}$ is chosen as:

$$
\zeta_{\text {ext }}=\left\{\begin{array}{l}
0 \\
0.875 .
\end{array}\right.
$$

In the first case (figures 1 and 2) the initial Gaussian "wall of charge" evolves into two mirror-image "walls of charge" that propagate with equal Newtonian speed in opposite directions and tend towards the speed of light as $t \rightarrow \infty$. In the second case (figures [3] and 4) the initial Gaussian "wall of charge" is accelerated by a combination of the external field and its self-field and approximately maintains its shape. 


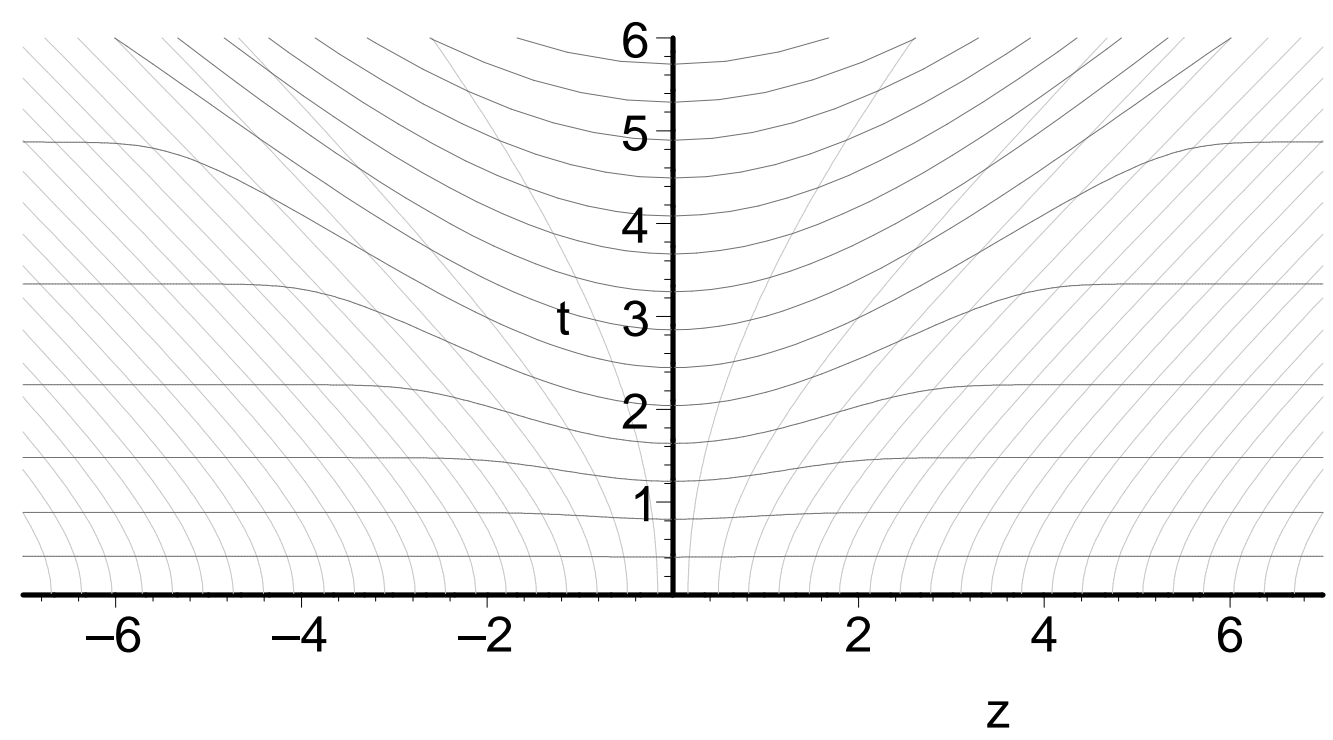

Figure 2: The lines of constant $\tau$ and $\sigma$ generated by the initial charge distribution (69) with zero applied external field. Lines of constant $\tau$, except $\tau=0$, are dark grey and lines of constant $\sigma$ (the streamlines of the flow), except $\sigma=0$, are light grey. The black line $\tau=0$ coincides with the $z$-axis and the black line $\sigma=0$ coincides with the $t$-axis.

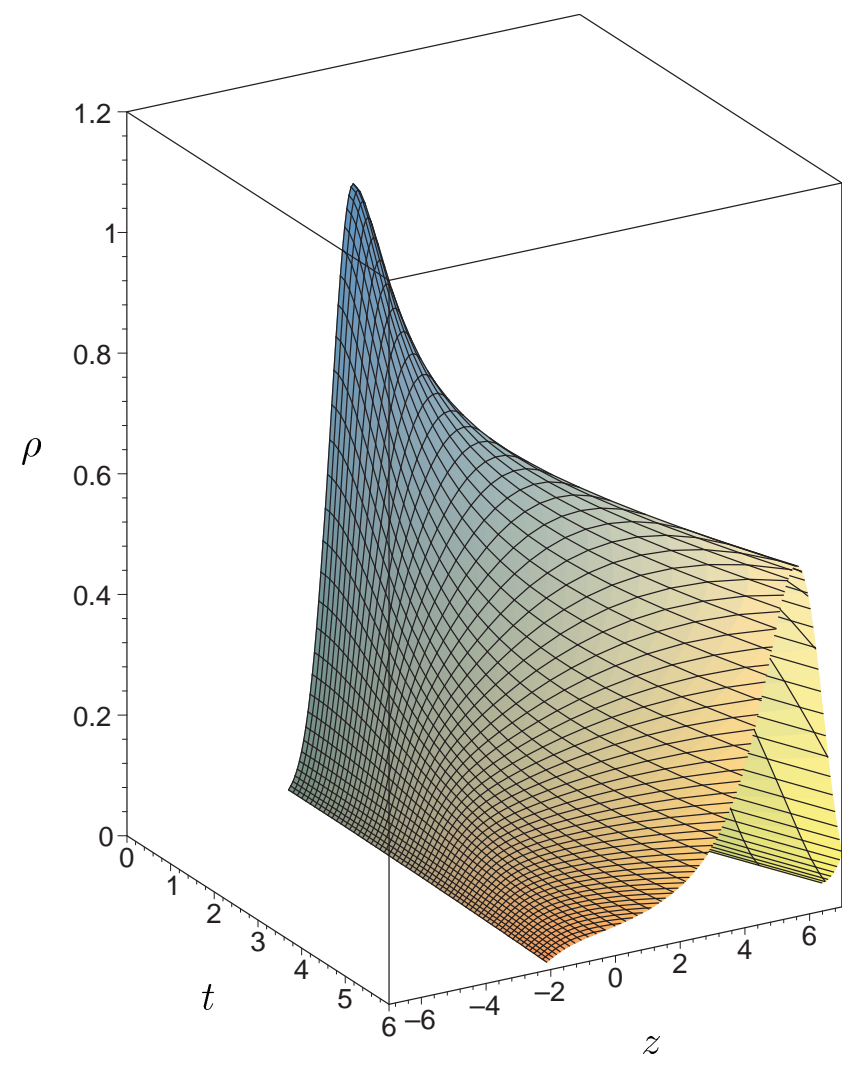

Figure 3: The time history of the initial charge distribution (69) with finite applied external field. 


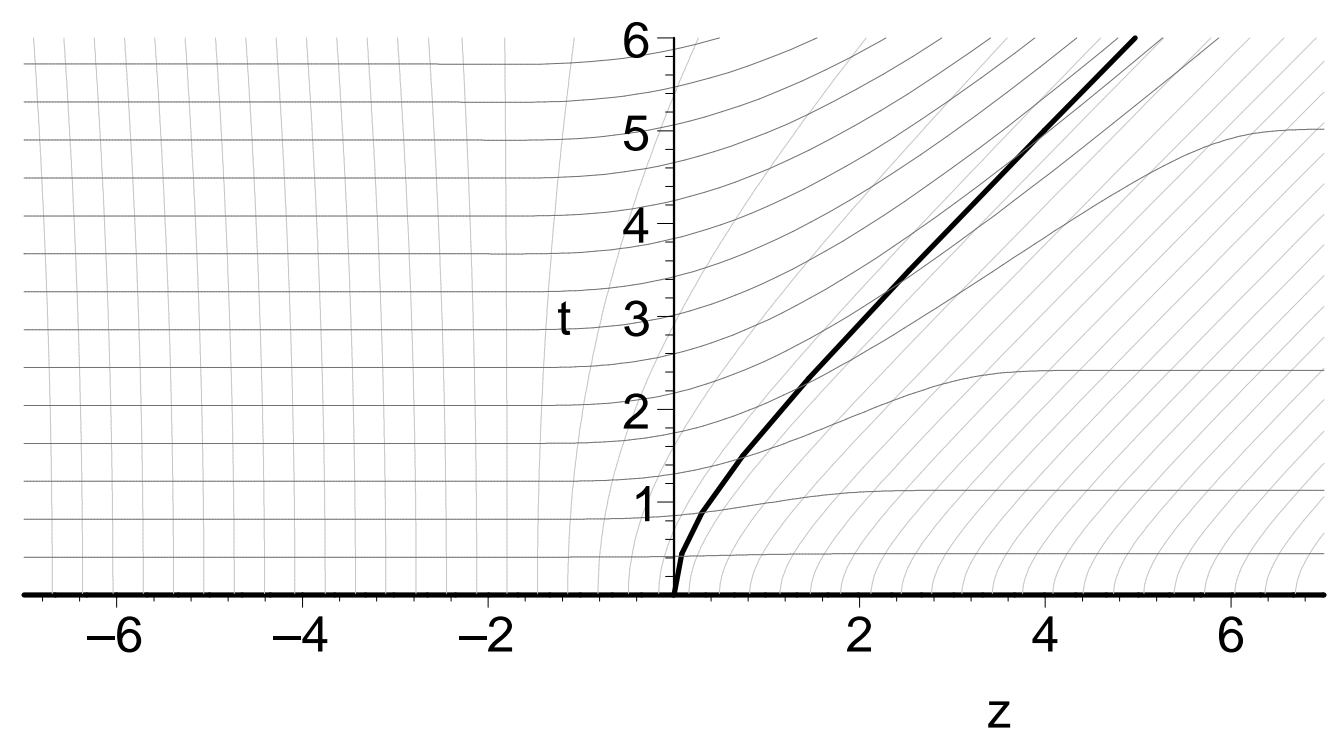

Figure 4: The lines of constant $\tau$ and $\sigma$ generated by the initial charge distribution (69) with finite applied external field. Lines of constant $\tau$, except $\tau=0$, are dark grey and lines of constant $\sigma$ (the streamlines of the flow), except $\sigma=0$, are light grey. The black line $\tau=0$ coincides with the $z$-axis and the black curve lying in the region $z>0$ is $\sigma=0$.

\subsection{Laboratory frame description}

Although the solutions above are exact in terms of $(\tau, \sigma)$, solving (67) for $\hat{\sigma}(t, z)$ in closed form is impossible if $\zeta$ is arbitrary. One way of tackling this problem is to introduce a running parameter $\varepsilon>0$, replace $\hat{\sigma}$ by a 1-parameter family of functions $\hat{\sigma}^{\varepsilon}$ and solve (67) for $\hat{\sigma}^{\varepsilon}$ order-by-order in $\varepsilon$.

To effect the coordinate transformation from $(\tau, \sigma)$ to $(t, z) \zeta$ is replaced with the 1-parameter family of functions $\zeta^{\varepsilon}$ :

$$
\zeta^{\varepsilon}(\sigma)=\frac{1}{\varepsilon} \zeta_{-1}+\zeta_{0}(\sigma) .
$$

where $\zeta_{\text {ext }}^{\varepsilon}=\frac{1}{\varepsilon} \zeta_{-1} \neq 0$ and $\zeta_{\text {self }}=\zeta_{0}$. One may interpret (70) by saying that external field effects (the first term $\frac{1}{\varepsilon} \zeta_{-1}$ ) dominate the effects of space-charge (the second term $\zeta_{0}$ ). The coefficients of the $\varepsilon$ terms in (70) have been labelled by the $\varepsilon$-order of the term in which they appear; this convention will be used consistently throughout this article.

A solution to (67) can be obtained order-by-order in $\varepsilon$ using the series ansatz for $t>0$

$$
\hat{\sigma}^{\varepsilon}(t, z)=\sum_{n=0}^{\infty} \varepsilon^{n} \hat{\sigma}_{n}(t, z) .
$$

The electric field component $\mathcal{E}^{\varepsilon}(t, z)$ and 4-velocity field $V^{\varepsilon}$ can be written entirely in terms of $\hat{\sigma}^{\varepsilon}, t$ and $z$ :

$$
\begin{aligned}
& \mathcal{E}^{\varepsilon}(t, z)=\zeta^{\varepsilon}\left(\hat{\sigma}^{\varepsilon}(t, z)\right) \\
& V^{\varepsilon}=\sqrt{1+\left[\zeta^{\varepsilon}\left(\hat{\sigma}^{\varepsilon}(t, z)\right)\right]^{2} t^{2}} \partial_{t}+\zeta^{\varepsilon}\left(\hat{\sigma}^{\varepsilon}(t, z)\right) t \partial_{z} .
\end{aligned}
$$


Using (70), (67), (71), the choice $\zeta_{-1}>0$ and $t>0$, by equating orders in $\varepsilon$ it follows that

$$
\begin{aligned}
& \hat{\sigma}^{\varepsilon}(t, z)=z-t+\frac{\varepsilon}{\zeta_{-1}}-\frac{1+2 t \zeta_{0}(z-t)}{2 t \zeta_{-1}^{2}} \varepsilon^{2}+O\left(\varepsilon^{3}\right) \\
& \mathcal{E}^{\varepsilon}(t, z)=\frac{1}{\varepsilon} \zeta_{-1}+\zeta_{0}(z-t)+\frac{\zeta_{0}^{\prime}(z-t)}{\zeta_{-1}} \varepsilon+O\left(\varepsilon^{2}\right) \\
& V^{\varepsilon}=\left[\frac{1}{\varepsilon} \zeta_{-1}+\zeta_{0}(z-t)\right] t\left(\partial_{t}+\partial_{z}\right)+\left[\frac{1+2 t^{2} \zeta_{0}^{\prime}(z-t)}{2 t \zeta_{-1}} \partial_{t}+\frac{\zeta_{0}^{\prime}(z-t) t}{\zeta_{-1}} \partial_{z}\right] \varepsilon+O\left(\varepsilon^{2}\right)
\end{aligned}
$$

where $\zeta_{0}^{\prime}(z) \equiv \frac{d \zeta_{0}}{d z}(z)$. Although $V^{\varepsilon} \cdot V^{\varepsilon}=-1$ the $(t, z)$ components of $V^{\varepsilon}$ diverge in the limits $t \rightarrow \infty$ and $t \rightarrow 0$. Divergences as $t \rightarrow \infty$ are expected because the dominant term in the electric field is constant, and so the charged continuum is undergoing constant acceleration to leading order in $\varepsilon$. Although $V^{\varepsilon}$ diverges as $t \rightarrow \infty$, the electric current $J^{\varepsilon} \equiv \rho^{\varepsilon} V^{\varepsilon}$ is

$$
\begin{aligned}
J^{\varepsilon} & =\rho^{\varepsilon} V^{\varepsilon}=\widetilde{\# d \mathcal{E}^{\varepsilon}} \\
& =\zeta_{0}^{\prime}(z-t)\left(\partial_{t}+\partial_{z}\right)+\varepsilon \frac{\zeta_{0}^{\prime \prime}(z-t)}{\zeta_{-1}}\left(\partial_{t}+\partial_{z}\right)+O\left(\varepsilon^{2}\right),
\end{aligned}
$$

where (57) has been used. Since $J^{\varepsilon}=\rho^{\varepsilon} V^{\varepsilon}, J^{\varepsilon}=O\left(\varepsilon^{0}\right)$ and $V^{\varepsilon}=O\left(\varepsilon^{-1}\right)$ it follows that the reduced proper charge density $\rho^{\varepsilon}=O(\varepsilon)$. The leading order contribution to $\rho^{\varepsilon}$ is obtained by comparing (75) with (74) and using $J_{0}=\rho_{1} V_{-1}$ where

$$
J^{\varepsilon}=\sum_{n=0}^{\infty} \varepsilon^{n} J_{n}, \quad V^{\varepsilon}=\sum_{n=-1}^{\infty} \varepsilon^{n} V_{n}, \quad \rho^{\varepsilon}=\sum_{n=1}^{\infty} \varepsilon^{n} \rho_{n}
$$

It follows that ${ }^{5}$

$$
\rho^{\varepsilon}=\varepsilon \frac{\zeta_{0}^{\prime}}{\zeta_{-1} t}+O\left(\varepsilon^{2}\right)
$$

Equation (75) shows that $J^{\varepsilon}$ is bounded to $O\left(\varepsilon^{2}\right)$ as $t \rightarrow \infty$. The divergence as $t \rightarrow 0$ of the coefficient of $\varepsilon$ in equation (74) stems from the square-root term in (67). Evidently, the domain on which the approximate solution is valid (the half-plane $t>0$ ) is a subset of the domain of the exact solution (the entire $(t, z)$ plane). This is not too surprising since the charged continuum is at rest at $t=0$ and the leading term $J_{0}$ in the series for $J^{\varepsilon}$ is light-like:

$$
J_{0} \cdot J_{0}=0
$$

There is no observer frame in which $J_{0}$ is instantaneously at rest.

This simple example suggests that the series

$$
F^{\varepsilon}=\sum_{n=-1}^{\infty} \varepsilon^{n} F_{n}, \quad V^{\varepsilon}=\sum_{n=-1}^{\infty} \varepsilon^{n} V_{n}, \quad \rho^{\varepsilon}=\sum_{n=1}^{\infty} \varepsilon^{n} \rho_{n}
$$

where $F_{-1}$ is an external field (a solution to the source-free Maxwell equations), should be inserted into the field system (52,55) which is then solved order-by-order in $\varepsilon$.

\footnotetext{
${ }^{4}$ Similar solutions are obtained for $\zeta_{-1}<0$ but with $z+t$ replacing $z-t$.

${ }^{5}$ It should be noted that the equation $\rho^{\varepsilon}=-J^{\varepsilon} \cdot V^{\varepsilon}$ cannot be used to calculate (77) without first calculating $J_{2}$ since $\rho_{1}=-\left(J_{2} \cdot V_{-1}+J_{1} \cdot V_{0}+J_{0} \cdot V_{1}\right)$.
} 
This approach can lead to the series expansions for the highly symmetric solutions just discussed, but can also yield $\varepsilon$ expansions for solutions that depend on $(x, y)$ as well as $(t, z)$. A consequence of the above series expansions is that the electric 4-current has the form

$$
J^{\varepsilon}=\rho^{\varepsilon} V^{\varepsilon}=\sum_{n=0}^{\infty} \varepsilon^{n} J_{n} .
$$

It will be shown below that the above expansions partially decouple the field system (52 55) yielding an infinite hierarchy of equations that are amenable to solution when supplemented with appropriate boundary conditions and initial data.

\section{The perturbation scheme}

Based on the above exact symmetric solution it is assumed that there exists a class of solutions to (52,55) that represents configurations of charged particles in ultra-relativistic collective motion. It is shown in Appendix A.1 that the notion of an ultra-relativistic 4-velocity vector field can be made precise and should be distinguished from the magnitude of a relative Newtonian speed that can be defined for any two time-like 4-vector fields. An ultra-relativistic velocity field is a pointwise limiting concept that depends on the existence of the forward light-cone structure at each event in spacetime. To leading order in the expansion defined below the velocity field of the charged continuum is light-like. The full series will be considered as an asymptotic expansion for a solution to (52,55) and physically represents an ultra-relativistic configuration controlled by the parameter $\varepsilon$. Such configurations cannot of course be exhaustive. They are chosen to be representative of the class relevant to charged beams in high-energy accelerators.

Introduce perturbation series for $\left(V^{\varepsilon}, \rho^{\varepsilon}, F^{\varepsilon}\right)$ in $\varepsilon$ of the form

$$
V^{\varepsilon}=\sum_{n=-1}^{\infty} \varepsilon^{n} V_{n}, \quad \rho^{\varepsilon}=\sum_{n=1}^{\infty} \varepsilon^{n} \rho_{n}, \quad F^{\varepsilon}=\sum_{n=-1}^{\infty} \varepsilon^{n} F_{n}
$$

where

$$
V_{n} \in \Gamma T \mathcal{M}, \quad \rho_{n} \in \Gamma \Lambda^{0} \mathcal{M}, \quad F_{n} \in \Gamma \Lambda^{2} \mathcal{M}
$$

Theorem 1. Using (52,56) the coefficients of the expansions in (78) satisfy

$$
\begin{aligned}
& d F_{n-1}=0 \quad \text { for } n \in\{0,1,2, \ldots\} \\
& d \star F_{n-1}= \begin{cases}0 & \text { for } n=0 \\
-\sum_{r=1}^{n} \star \rho_{r} \widetilde{V}_{n-r-1} & \text { for } n \in\{1,2, \ldots\}\end{cases} \\
& \sum_{r=0}^{n} \nabla_{V_{r-1}} \widetilde{V}_{n-r-1}=\sum_{r=0}^{n} i_{V_{r-1}} F_{n-r-1} \quad \text { for } n \in\{0,1,2, \ldots\} \\
& \sum_{r=0}^{n} V_{r-1} \cdot V_{n-r-1}= \begin{cases}-1 & \text { for } n=2 \\
0 & \text { for } n \in\{0,1,3,4,5, \ldots\}\end{cases} \\
& \sum_{r=1}^{n+1} d \star\left(\rho_{r} \widetilde{V}_{n-r}\right)=0 \quad \text { for } n \in\{0,1,2, \ldots\}
\end{aligned}
$$


The partially decoupled equations (80,84) are amenable to an ordered analysis and can be arranged into the hierarchy:

For $N \in\{0,1,2, \ldots\}$ :

Step $3 N+1$ : Solve (80) and (81) with $n=N$ for $F_{n-1}$.

Step $3 N+2$ : Solve (82) and (83) with $n=N$ for $V_{n-1}$.

Step $3 N+3$ : Solve (84) with $n=N$ for $\rho_{n+1}$.

Proof. Equations (80,84) are obtained by inserting the series (78) into (52,55) and the integrability condition (56). The hierarchical structure follows by inspection.

To illustrate the partially decoupled structure in (85) consider the first 8 steps in detail:

Step 1: Adopt an external electromagnetic field $F_{-1}$ i.e. a solution of the source-free equations

$$
d F_{-1}=0 \quad \text { and } \quad d \star F_{-1}=0 .
$$

Step 2: Solve

$$
\nabla_{V_{-1}} \widetilde{V}_{-1}=i_{V_{-1}} F_{-1} \quad \text { subject to } \quad V_{-1} \cdot V_{-1}=0
$$

for $V_{-1} \neq 0$, where $F_{-1}$ is data obtained in the previous step.

Although (87) is non-linear in the unknown field $V_{-1}$ it is straightforward to analyse; it can be written as a quasi-linear second-order ordinary differential equation for the integral curves of $V_{-1}$ and is the only non-linear differential equation in this scheme.

It may seem that the pair of equations above offer 5 scalar equations for 4 unknown scalars. However, the contraction of the first equation with $V_{-1}$ identically vanishes, so there are only 4 independent equations. This can be seen $^{6}$ by writing (87) with respect to an arbitrary basis and noting that one of the 5 components of (87) is a consequence of the other 4 .

Step 3: The leading order reduced proper charge density $\rho_{1}$ is a solution to

$$
d \star\left(\rho_{1} \widetilde{V}_{-1}\right)=0 .
$$

Step 4: The 2-form $F_{0}$ is a solution to Maxwell's equations with the current $\rho_{1} \widetilde{V}_{-1}$ as a source:

$$
d F_{0}=0 \quad \text { and } \quad d \star F_{0}=-\star \rho_{1} \widetilde{V}_{-1} .
$$

The conservation equation (88) ensures that (89) has a solution.

Step 5: The zero order velocity field $V_{0}$ is obtained from the linear equations

$$
\nabla_{V_{-1}} \widetilde{V}_{0}+\nabla_{V_{0}} \widetilde{V}_{-1}=i_{V_{-1}} F_{0}+i_{V_{0}} F_{-1} \quad \text { subject to } \quad V_{-1} \cdot V_{0}=0
$$

Step 6: The second order coefficient $\rho_{2}$ in the $\varepsilon$ expansion for the reduced proper charge density $\rho^{\varepsilon}$ satisfies

$$
d \star\left(\rho_{2} \widetilde{V}_{-1}\right)+d \star\left(\rho_{1} \widetilde{V}_{0}\right)=0
$$

${ }^{6}$ The split of (87) with respect to the laboratory frame is given in Appendix A.2 The equation obtained by contracting (87) with the laboratory observer field $\partial_{t}$ is redundant because it follows as a consequence of (119) and (120). 
Step 7: Equation (91) ensures that

$$
d F_{1}=0 \quad \text { and } \quad d \star F_{1}=-\star \rho_{2} \widetilde{V}_{-1}-\star \rho_{1} \widetilde{V}_{0}
$$

may be solved for the coefficient $F_{1}$ (the first order term in the $\varepsilon$ expansion of $F^{\varepsilon}$ ) given appropriate initial and boundary conditions.

Step 8: The linear partial differential equation for $V_{1}$ is

$$
\nabla_{V_{-1}} \widetilde{V}_{1}+\nabla_{V_{0}} \widetilde{V}_{0}+\nabla_{V_{1}} \widetilde{V}_{-1}=i_{V_{-1}} F_{1}+i_{V_{0}} F_{0}+i_{V_{1}} F_{-1} \quad \text { subject to } \quad 2 V_{-1} \cdot V_{1}+V_{0} \cdot V_{0}=-1
$$

$V^{\varepsilon}$ must be calculated to at least first order in $\varepsilon$ to obtain a time-like vector field. To see this let $X^{\varepsilon}=\varepsilon^{-1} V_{-1}+V_{0}$ and note $X^{\varepsilon} \cdot X^{\varepsilon}=V_{0} \cdot V_{0}$ using the metric-product conditions in (87) and (90). Using lemma 5 in Appendix A.1 $V_{0} \cdot V_{0} \geq 0$ and so $X^{\varepsilon}$ is light-like or space-like. Now introduce $Y^{\varepsilon}=X^{\varepsilon}+\varepsilon V_{1}=\varepsilon^{-1} V_{-1}+V_{0}+\varepsilon V_{1}$ and note $Y^{\varepsilon} \cdot Y^{\varepsilon}=V_{0} \cdot V_{0}+2 V_{1} \cdot V_{-1}+O(\varepsilon)=-1+O(\varepsilon)$ using (93). Therefore, there exists some value of $\varepsilon>0$ for which $Y^{\varepsilon}$ is time-like.

It has already been stated that the pair of equations (87) in step 2 may be considered as 4 non-linear equations in 4 unknowns. Furthermore:

Lemma 2. The equations in each step $3 N+2$, for $N \geq 1$, are equivalent to a set of 4 independent inhomogeneous linear equations for the 4 unknown components of $V_{N-1}$.

Proof. Step $3 N+2$ involves solving (82) and (83) for $V_{N-1}$. Assume that the equations in steps 1 to $3 N+1$ have been solved and define $L_{n}$ as

$$
L_{n}=\sum_{r=0}^{n}\left(\nabla_{V_{n-r-1}} \widetilde{V}_{r-1}-i_{V_{r-1}} F_{n-r-1}\right) .
$$

Clearly (82) is equivalent to $L_{n}=0$. The vector fields $V_{-1}, \ldots, V_{N-2}$ have been obtained in steps 1 to $3 N+1$ and so $L_{n}=0$ for $n=0, \ldots, N-1$. It will now be shown that given any $V_{N-1}$ satisfying (83) with $n=N$ the scalar field $i_{V_{-1}} L_{N}$ identically vanishes.

From (83) for $n=0, \ldots, N$

$$
\begin{aligned}
0=\nabla_{V_{s}}\left(\sum_{r=0}^{n} V_{r-1} \cdot V_{n-r-1}\right) & =\sum_{r=0}^{n}\left(\nabla_{V_{s}} V_{r-1}\right) \cdot V_{n-r-1}+\sum_{r=0}^{n} V_{r-1} \cdot \nabla_{V_{s}} V_{n-r-1} \\
& =\sum_{r=0}^{n}\left(\nabla_{V_{s}} V_{r-1}\right) \cdot V_{n-r-1}+\sum_{q=n}^{0} V_{n-q-1} \cdot \nabla_{V_{s}} V_{q-1}
\end{aligned}
$$

where the second sum has been re-labelled by $q=n-r$. The two sums are identical so

$$
\sum_{r=0}^{n}\left(\nabla_{V_{s}} V_{n-r-1}\right) \cdot V_{r-1}=0
$$


Using (94) and $L_{n}=0$ for all $0 \leq n<N$

$$
\begin{aligned}
i_{V_{-1}} L_{N} & =\sum_{n=0}^{N} i_{V_{n-1}} L_{N-n} \\
& =\sum_{n=0}^{N} i_{V_{n-1}} \sum_{r=0}^{N-n}\left(\nabla_{V_{N-n-r-1}} \widetilde{V}_{r-1}-i_{V_{r-1}} F_{N-n-r-1}\right) \\
& =\sum_{n=0}^{N} \sum_{r=0}^{N-n}\left(V_{n-1} \cdot \nabla_{V_{N-n-r-1}} V_{r-1}-i_{V_{n-1}} i_{V_{r-1}} F_{N-n-r-1}\right) \\
& =\sum_{n=0}^{N} \sum_{s=n}^{N}\left(V_{n-1} \cdot \nabla_{V_{N-s-1}} V_{s-n-1}-i_{V_{n-1}} i_{V_{s-n-1}} F_{N-s-1}\right)
\end{aligned}
$$

where, in the final step, the innermost sum has been re-labelled by $s=n+r$. The double summation in (96) is over the set

$$
\{(n, s) \mid n, s \in\{0,1, \ldots, N\}, n \leq s\}
$$

and so

$$
\sum_{n=0}^{N} \sum_{s=n}^{N} S_{n s}=\sum_{s=0}^{N} \sum_{n=0}^{s} S_{n s}
$$

for any summand $S_{n s}$. Therefore, using (96)

$$
\begin{aligned}
i_{V_{-1}} L_{N} & =\sum_{s=0}^{N}\left(\sum_{n=0}^{s} V_{n-1} \cdot \nabla_{V_{N-s-1}} V_{s-n-1}-\sum_{n=0}^{s} i_{V_{n-1}} i_{V_{s-n-1}} F_{N-s-1}\right) \\
& =0
\end{aligned}
$$

where the first term in the summand vanishes because of (95) and the second term vanishes because $i_{V_{n-1}} i_{V_{s-n-1}}=-i_{V_{s-n-1}} i_{V_{n-1}}$ and the sum over $s$ is from 0 to $n$.

Note that the lemma applies to step 5 and beyond and so, for the purposes of the lemma, the fields $F_{-1}, V_{-1}, \rho_{1}, F_{0}$ obtained in steps 1 to 4 are prescribed data. Let $\left\{X_{1}, X_{2}, X_{3}, X_{4}\right\}$, with $X_{i} \in \Gamma T \mathcal{M}$ for $i=1,2,3,4$, be any vector frame such that $X_{1}=V_{-1}$ and define $\left\{e^{1}, e^{2}, e^{3}, e^{4}\right\}$, where $e^{i} \in \Gamma \Lambda^{1} \mathcal{M}$, to be the coframe dual to $\left\{X_{1}, X_{2}, X_{3}, X_{4}\right\}$ :

$$
e^{i}\left(X_{j}\right)=\delta_{j}^{i}
$$

where $\delta_{j}^{i}$ is the Kronecker delta. Equation (82) can be written as

$$
\mathcal{L}\left(\widetilde{V}_{N-1}\right)=\xi_{N-1}
$$

where $\mathcal{L}$ is the $\mathbb{R}$-linear differential operator on $\Gamma \Lambda^{1} \mathcal{M}$

$$
\mathcal{L}\left(\widetilde{V}_{N-1}\right)=\nabla_{V_{-1}} \widetilde{V}_{N-1}+\nabla_{V_{N-1}} \widetilde{V}_{-1}+i_{V_{N-1}} F_{-1}
$$

where $\xi_{N-1} \in \Gamma \Lambda^{1} \mathcal{M}$ depends on $\left\{V_{-1}, V_{0} \ldots, V_{N-2}, F_{0}, \ldots, F_{N-1}\right\}$ but is independent of $V_{N-1}$. Hence, using (97)

$$
i_{X_{1}}\left(\mathcal{L}\left(\widetilde{V}_{N-1}\right)-\xi_{N-1}\right)=i_{V_{-1}}\left(\mathcal{L}\left(\widetilde{V}_{N-1}\right)-\xi_{N-1}\right)=0
$$


Likewise, equation (83) can be expressed as

$$
i_{X_{1}} \widetilde{V}_{N-1}=i_{V_{-1}} \widetilde{V}_{N-1}=C_{N-1}
$$

where the scalar field $C_{N-1} \in \Gamma \Lambda^{0} \mathcal{M}$ depends only on $\left\{V_{0}, \ldots, V_{N-2}\right\}$.

Using the coframe $\left\{e^{1}, e^{2}, e^{3}, e^{4}\right\}$

$$
\widetilde{V}_{N-1}=\sum_{i=1}^{4} \lambda_{N-1, i} e^{i}
$$

for component fields $\lambda_{N-1, i}$. From (100) $\lambda_{N-1,1}=C_{N-1}$ and (98) becomes

$$
\mathcal{L}\left(\sum_{i=2}^{4} \lambda_{N-1, i} e^{i}\right)=\xi_{N-1}-\mathcal{L}\left(C_{N-1} e^{1}\right)
$$

From (99) $i_{X_{1}}$ annihilates (101) identically and

$$
i_{X_{j}}\left(\mathcal{L}\left(\sum_{i=2}^{4} \lambda_{N-1, i} e^{i}\right)\right)=i_{X_{j}}\left(\xi_{N-1}-\mathcal{L}\left(C_{N-1} e^{1}\right)\right) \quad \text { for } \quad j=2,3,4 .
$$

Thus, using the inhomogeneous linear algebraic constraint (83), 3 inhomogeneous linear differential equations have been obtained for the 3 unknown fields $\left\{\lambda_{N-1,2}, \lambda_{N-1,3}, \lambda_{N-1,4}\right\}$ and $\lambda_{N-1,1}$ is obtained using (83), determining $V_{N-1}$.

In conclusion, the only non-linear equations in the hierarchy developed in theorem 1 are those for $V_{-1}$. All of the remaining equations are linear and therefore, in principle, simpler to solve.

\section{Example : Charged beam propagating in free space}

As a simple application of the hierarchy (80,84) consider a high-energy charged beam propagating in free space. Unlike the exact "wall of charge" solutions derived earlier, the charge distributions considered here have finite extent transverse to and along the direction of propagation. The first eight steps of the hierarchy (80,84) are:

Step 1: The leading order Maxwell field $F_{-1}$ (the external field) is chosen to vanish so (866) is trivially satisfied.

Step 2: Using (87) the leading order 4-velocity field $V_{-1}$ satisfies

$$
\nabla_{V_{-1}} V_{-1}=0, \quad V_{-1} \cdot V_{-1}=0
$$

since the external field $F_{-1}=0$. Therefore, consider solutions to (80,84) adapted to a null geodesic coordinate system $(u, v, x, y)$ where $u=z-t, v=z+t$ and

$$
\begin{aligned}
g & =-d t \otimes d t+d x \otimes d x+d y \otimes d y+d z \otimes d z \\
& =\frac{1}{2}(d u \otimes d v+d v \otimes d u)+d x \otimes d x+d y \otimes d y
\end{aligned}
$$


with the spacetime volume 4 -form

$$
\star 1=d t \wedge d z \wedge \#_{\perp} 1
$$

where

$$
\# \perp 1 \equiv d x \wedge d y
$$

The pair $\left(\partial_{t}, \partial_{z}\right)$ and their duals $(d t, d z)$ are related to $\left(\partial_{v}, \partial_{u}\right)$ and $(d v, d u)$ as follows:

$$
\begin{aligned}
\partial_{v} & =\frac{1}{2}\left(\partial_{z}+\partial_{t}\right), \quad \partial_{u}=\frac{1}{2}\left(\partial_{z}-\partial_{t}\right), \\
d v & =d z+d t, \quad d u=d z-d t .
\end{aligned}
$$

Metric identities used in the subsequent steps are

$$
\star(d u \wedge \alpha)=(-1)^{p} d u \wedge \#_{\perp} \alpha, \quad \star(d v \wedge \alpha)=-(-1)^{p} d v \wedge \#_{\perp} \alpha
$$

where $\#_{\perp}(\alpha \wedge \tilde{X})=i_{X} \#_{\perp} \alpha$ and $\alpha$ is any $p$-form such that $i_{\partial_{u}} \alpha=i_{\partial_{v}} \alpha=0$. The vector field $V_{-1}$ is chosen as

$$
\begin{aligned}
V_{-1} & =\gamma_{-1}\left(\partial_{t}+\partial_{z}\right) \\
& =2 \gamma_{-1} \partial_{v}
\end{aligned}
$$

where $\gamma_{-1} \neq 0$ is assumed and, using $\nabla \partial_{v}=\nabla \partial_{u}=0$, the only non-zero component of (102) is

$$
\partial_{v} \gamma_{-1}=0
$$

Hence, $\gamma_{-1}$ is independent of $v$.

Step 3: Using (105), equation (88) is

$$
\partial_{v}\left(\rho_{1} \gamma_{-1}\right)=0
$$

and so, using (106)

$$
\partial_{v} \rho_{1}=0
$$

It follows that $\rho_{1}$ is independent of $v$.

Step 4: Using (104), particular solutions to (89) may be written

$$
F_{0}=d \Phi_{0} \wedge d u
$$

where the scalar potential $\Phi_{0}$ is chosen to satisfy

$$
\partial_{v} \Phi_{0}=0, \quad d_{\perp} \#_{\perp} d_{\perp} \Phi_{0}=\rho_{1} \gamma_{-1} \#_{\perp} 1
$$

and $d_{\perp}$ is the exterior derivative in the $(x, y)$ plane defined by

$$
d_{\perp} f=\partial_{x} f d x+\partial_{y} f d y
$$

for any 0 -form $f \in \Gamma \Lambda^{0} \mathcal{M}$. 
Step 5: Using (90), the equations for $V_{0}$ are

$$
\nabla_{V_{-1}} \tilde{V}_{0}+\nabla_{V_{0}} \tilde{V}_{-1}=i_{V_{-1}} F_{0}, \quad V_{-1} \cdot V_{0}=0
$$

and are solved by the particular solution

$$
V_{0}=0
$$

since $i_{V_{-1}} F_{0}=0$ using (107), (108) and (105).

Step 6: Since $V_{0}=0$ it follows that (91) has the form

$$
d \star\left(\rho_{2} \tilde{V}_{-1}\right)=0
$$

and, using (105) and (106), is solved by any $\rho_{2}$ satisfying

$$
\partial_{v} \rho_{2}=0
$$

i.e. $\rho_{2}$ is independent of $v$.

Step 7: Since $V_{0}=0$ the Maxwell equations (92) are

$$
d F_{1}=0, \quad d \star F_{1}=-\rho_{2} \star \tilde{V}_{-1}
$$

and, employing the same method used in step 4, are solved by the particular solution

$$
F_{1}=d \Phi_{1} \wedge d u
$$

where $\Phi_{1}$ is chosen to satisfy

$$
\partial_{v} \Phi_{1}=0, \quad d_{\perp} \#_{\perp} d_{\perp} \Phi_{1}=\rho_{2} \gamma_{-1} \#_{\perp} 1 .
$$

Step 8: Using (93), the equations for $V_{1}$ are

$$
\nabla_{V_{-1}} \tilde{V}_{1}+\nabla_{V_{1}} \tilde{V}_{-1}=i_{V_{-1}} F_{1}, \quad V_{-1} \cdot V_{1}=-\frac{1}{2}
$$

and since $i_{V_{-1}} F_{1}=0$ (which follows using (109), (110) and (105) ) a particular solution to (111) is

$$
V_{1}=-\frac{1}{2 \gamma_{-1}} \partial_{u}, \quad \partial_{u} \gamma_{-1}=0
$$

using (106), (105) and $\nabla \partial_{v}=\nabla \partial_{u}=0$. Hence, the function $\gamma_{-1}$ is independent of $u$ and therefore, using (106),$\gamma_{-1}$ depends only on $x$ and $y$.

In summary these solutions, describing an ultra-relativistic charged distribution propagating along the $z$-axis with its electromagnetic self-fields, are

$$
\begin{aligned}
V^{\varepsilon} & =\frac{1}{\varepsilon} 2 \gamma_{-1} \partial_{v}-\varepsilon \frac{1}{2 \gamma_{-1}} \partial_{u}+O\left(\varepsilon^{2}\right) \\
& =\left(\frac{1}{\varepsilon} \gamma_{-1}+\frac{\varepsilon}{4 \gamma_{-1}}\right) \partial_{t}+\left(\frac{1}{\varepsilon} \gamma_{-1}-\frac{\varepsilon}{4 \gamma_{-1}}\right) \partial_{z}+O\left(\varepsilon^{2}\right) \\
F^{\varepsilon} & =d \Phi_{0} \wedge d u+\varepsilon d \Phi_{1} \wedge d u+O\left(\varepsilon^{2}\right) \\
& =-\left(d \Phi_{0}+\varepsilon d \Phi_{1}\right) \wedge d t+\left(d \Phi_{0}+\varepsilon d \Phi_{1}\right) \wedge d z+O\left(\varepsilon^{2}\right)
\end{aligned}
$$


where $\Phi_{0}$ and $\Phi_{1}$ satisfy

$$
d_{\perp} \#_{\perp} d_{\perp} \Phi_{0}=\gamma_{-1} \rho_{1} \#_{\perp} 1, \quad d_{\perp} \#_{\perp} d_{\perp} \Phi_{1}=\gamma_{-1} \rho_{2} \#_{\perp} 1
$$

and

$$
\begin{aligned}
& \Phi_{0}=\hat{\Phi}_{0}(u, x, y), \quad \Phi_{1}=\hat{\Phi}_{1}(u, x, y) \\
& \rho_{1}=\hat{\rho}_{1}(u, x, y), \quad \rho_{2}=\hat{\rho}_{2}(u, x, y), \\
& \gamma_{-1}=\hat{\gamma}_{-1}(x, y)
\end{aligned}
$$

with $u=z-t$. The scalar fields $\rho_{0}, \rho_{1}, \gamma_{-1}$ are determined by their values on the space-like hypersurface $t=0$ given as data. The potentials $\Phi_{0}$ and $\Phi_{1}$ are then solved in terms of $\gamma_{-1} \rho_{0}$ and $\gamma_{-1} \rho_{1}$ using the 2-dimensional Poisson equations (112) in the $(x, y)$ plane.

The 3-velocity of the beam is along the direction $z$ in the laboratory frame with Newtonian speed

$$
\frac{\frac{1}{\varepsilon} \gamma_{-1}-\varepsilon \frac{1}{4 \gamma_{-1}}+O\left(\varepsilon^{2}\right)}{\frac{1}{\varepsilon} \gamma_{-1}+\varepsilon \frac{1}{4 \gamma_{-1}}+O\left(\varepsilon^{2}\right)}=1-\frac{\varepsilon^{2}}{2 \gamma_{-1}^{2}}+O\left(\varepsilon^{3}\right) .
$$

For example, consider a Gaussian bunch with transverse radius $R_{0}$ travelling at constant Newtonian speed $1-\frac{\varepsilon^{2}}{2 b_{0}^{2}}$ to order $\varepsilon^{2}$ :

$$
\hat{\gamma}_{-1}(x, y)=b_{0}, \quad \hat{\rho}_{1}(z, x, y)=a_{0} \exp \left(-\frac{x^{2}+y^{2}}{R_{0}^{2}}\right) \Xi(z)
$$

where $a_{0}, R_{0}$ and $b_{0}$ are constants and $\Xi: \mathbb{R} \rightarrow \mathbb{R}$ is a smooth bump function vanishing outside the interval $\left(-z_{1}, z_{1}\right)$ and $\Xi(z)=1$ for $z \in\left(-z_{2}, z_{2}\right)$ and $z_{1}>z_{2}>0$. Then the laboratory reduced charge density $\gamma_{-1} \rho_{1}$ for some range of $t$ is

$$
\gamma_{-1} \rho_{1}=\hat{\gamma}_{-1}(x, y) \hat{\rho}_{1}(z-t, x, y)=a_{0} b_{0} \exp \left(-\frac{x^{2}+y^{2}}{R^{2}}\right) \Xi(z-t) .
$$

Working in the cylindrical polar coordinates $(t, R, \phi, z)$ where $x=R \cos \phi$ and $y=R \sin \phi$, a cylindrically symmetric solution to (112) well-behaved at $R=0$ is

$$
\Phi_{0}=\left\{\int_{0}^{R} a_{0} b_{0} \frac{R_{0}^{2}}{2 s}\left[1-\exp \left(-\frac{s^{2}}{R_{0}^{2}}\right)\right] d s\right\} \Xi(z-t)
$$

and the corresponding electromagnetic 2 -form $F_{0}$ is

$$
F_{0}=a_{0} b_{0} \frac{R_{0}^{2}}{2 R}\left[1-\exp \left(-\frac{R^{2}}{R_{0}^{2}}\right)\right] \Xi(z-t) d R \wedge(-d t+d z) .
$$

The laboratory electric field is radial, the magnetic field is azimuthal and their magnitudes are equal and vanish outside of the support of $\Xi$.

\section{Conclusion}

When analysing the dynamics of charged particle beams it is often fruitful to adopt a description based on classical fields rather than classical point particles. Pathologies (such as pre-acceleration) associated with radiating point particles are avoided by relying on field-theoretical notions. 
A novel analysis of charged beam dynamics has been presented and a model of a freely propagating charged bunch discussed. The approach relies on an asymptotic series representation of solutions to self-consistent spacetime covariant field equations for $(V, \rho, F)$ describing a charged continuum. The asymptotic series for $V$ is based on the light-like vector field $V_{-1}$ leading to an ultra-relativistic approximation. The hierarchy of equations obtained are more amenable to analysis than the original non-linear field system and particular solutions have been presented.

There are numerous avenues for the development of this work involving ultra-relativistic charged beams in the vicinity of beam pipes, RF cavities, spoilers, etc. leading to dynamical effects that are often described in terms of "wake-fields" [8, 9]. This work will lead to a clearer understanding of radiation-reaction exhibited by continuum models of charged particle beams.

\section{Acknowledgements}

The authors acknowledge support from the Cockcroft Institute and the EU GIFT project (NESTAdventure Project no. 5006) and are grateful to Professor M Poole for enlightening discussions.

\section{References}

[1] L. D. Landau and E. M. Lifshitz, The Classical Theory of Fields (Pergamon, Oxford, 1962).

[2] E. Poisson, arXiv preprint gr-qc/9912045 (1990).

[3] F. Rohrlich, Classical Charged Particles (Addison-Wesley, Redwood City, 1990).

[4] J. D. Jackson, Classical Electrodynamics, 3rd ed., (Wiley, 1998).

[5] P. A. M. Dirac, Proc. Roy. Soc. London A 167, 148 (1938).

[6] C. Teitelboim, D. Villarroel and C. G. van Weert, Riv. Nuovo Cim. 3, 9, (1980).

[7] E. E. Flanagan and R. M. Wald, Phys. Rev. D 54, 6233 (1996).

[8] K. L. F. Bane, P. B. Wilson and T. Weiland, SLAC-PUB-3528, (1984).

[9] S. A. Heifets and S. A. Kheifets, Rev. Mod. Phys., 63, 3, 631-673, (1991).

[10] R. C. Davidson, Physics of Nonneutral Plasmas, (Addison-Wesley, 1990. Reissued by World Scientific, 2001).

[11] R. C. Davidson, Physics of Intense Charged Particle Beams, (World Scientific, 2001).

\section{A Appendix}

\section{A.1 Definition of ultra-relativistic and light-like limited vectors}

The spacetime description of a classical point particle with mass $m_{0}>0$ invokes a parametrised curve (world-line) with a future-pointing time-like tangent vector. For affinely parametrised curves the tangent vector $V$ (4-velocity) is normalised: $V \cdot V=-1$. A spacetime reference frame may be 
associated with a time-like vector field $U$ with $U \cdot U=-1$ and an "observer" in such a frame is modelled by an integral curve of $U$. The 4-momentum of the particle is defined to be $p=m_{0} V$. At an event where an observer curve intersects the world-line of the particle one has the orthogonal decomposition:

$$
p=\mathcal{E} U+\mathbf{P}
$$

where $U \cdot \mathbf{P}=0$. Relative to $U$, the scalar $\mathcal{E}$ is the energy of the particle (in units with $c=1$ ) and $\mathbf{P}$ is its 3-momentum. The Newtonian velocity $\mathbf{v}$ of the particle relative to $U$ is

$$
\mathbf{v}=\frac{\mathbf{P}}{\mathcal{E}}=-\frac{V}{V \cdot U}-U
$$

Since $U \cdot U=V \cdot V=-1$ implies $\mathcal{E}^{2}=\mathbf{P} \cdot \mathbf{P}+m_{0}^{2}$ it is traditional to say that the particle is "relativistic" relative to $U$ if $\mathcal{E}^{2} \simeq \mathbf{P} \cdot \mathbf{P}$ or equivalently $\mathbf{v} \cdot \mathbf{v} \simeq 1$. Clearly, such a notion depends on both $V$ and $U$ and the nearness of $\frac{\mathbf{P . P}}{\mathcal{E}^{2}}$ to unity. Furthermore, one may always find a frame in which $\mathbf{P}=0$ and $\mathcal{E}=m_{0}$. However, given a particular frame $U$ one may contemplate a 4-velocity $V$ that is relativistic relative to it. To generalise this notion for any frame one may consider a family of 4-velocities $V^{\varepsilon}$ and invoke properties of $V^{\varepsilon}$ as $\varepsilon$ tends to some limit.

Let $\varepsilon \in\left(0, \varepsilon_{\max }\right)=\left\{\varepsilon \in \mathbb{R} \mid 0<\varepsilon<\varepsilon_{\max }\right\}$ be the running parameter introduced in the main body of the article. A 1-parameter time-like vector field (which is not necessarily normalised) $W^{\varepsilon}$ is ultra-relativistic if for any time-like vector field $Z \in \Gamma T \mathcal{M}$

$$
\lim _{\varepsilon \rightarrow 0}\left(\frac{\left|W^{\varepsilon} \cdot Z\right|}{\left\|W^{\varepsilon}\right\|}\right)=\infty
$$

where $\|X\| \equiv \sqrt{-X \cdot X}$ for any time-like (or light-like) vector field $X \in \Gamma T \mathcal{M}$. Note that in this definition $Z$ is not a 1 -parameter vector field.

Lemma 3. Our definition of an ultra-relativistic vector field (114) on spacetime is independent of the choice of the time-like vector field $Z$.

Proof. Clearly (114) is independent of any rescaling of $Z$. Therefore let $Z$ and $\hat{Z}$ be two $g$-normalised future pointing time-like vector fields (i.e. $Z \cdot Z=\hat{Z} \cdot \hat{Z}=-1$ ) and define the vector field $X_{1}$ where

$$
X_{1}=\frac{(\hat{Z}+(\hat{Z} \cdot Z) Z)}{\left((\hat{Z} \cdot Z)^{2}-1\right)^{1 / 2}} .
$$

Hence

$$
\hat{Z}=-(\hat{Z} \cdot Z) Z+\left((\hat{Z} \cdot Z)^{2}-1\right)^{1 / 2} X_{1}
$$

and $X_{1} \cdot Z=0, X_{1} \cdot X_{1}=1$. Choose space-like unit vector fields $X_{2}, X_{3}$ so that $\left(Z, X_{1}, X_{2}, X_{3}\right)$ is a $g$-orthonormal frame. It follows that

$$
W^{\varepsilon} \cdot W^{\varepsilon}=-\left(W^{\varepsilon} \cdot Z\right)^{2}+\left(W^{\varepsilon} \cdot X_{1}\right)^{2}+\left(W^{\varepsilon} \cdot X_{2}\right)^{2}+\left(W^{\varepsilon} \cdot X_{3}\right)^{2}
$$

which implies

$$
\frac{\left(W^{\varepsilon} \cdot X_{1}\right)^{2}}{\left\|W^{\varepsilon}\right\|^{2}}=\frac{\left(W^{\varepsilon} \cdot Z\right)^{2}}{\left\|W^{\varepsilon}\right\|^{2}}-1-\frac{\left(W^{\varepsilon} \cdot X_{2}\right)^{2}}{\left\|W^{\varepsilon}\right\|^{2}}-\frac{\left(W^{\varepsilon} \cdot X_{3}\right)^{2}}{\left\|W^{\varepsilon}\right\|^{2}}
$$


and hence

$$
\frac{\left|W^{\varepsilon} \cdot X_{1}\right|}{\left\|W^{\varepsilon}\right\|} \leq \frac{\left|W^{\varepsilon} \cdot Z\right|}{\left\|W^{\varepsilon}\right\|} .
$$

The metric contraction of (115) with $\frac{W^{\varepsilon}}{\left\|W^{\varepsilon}\right\|}$ is

$$
\frac{\hat{Z} \cdot W^{\varepsilon}}{\left\|W^{\varepsilon}\right\|}=-(\hat{Z} \cdot Z) \frac{Z \cdot W^{\varepsilon}}{\left\|W^{\varepsilon}\right\|}+\left((\hat{Z} \cdot Z)^{2}-1\right)^{1 / 2} \frac{X_{1} \cdot W^{\varepsilon}}{\left\|W^{\varepsilon}\right\|}
$$

and so

$$
(\hat{Z} \cdot Z) \frac{Z \cdot W^{\varepsilon}}{\left\|W^{\varepsilon}\right\|}=-\frac{\hat{Z} \cdot W^{\varepsilon}}{\left\|W^{\varepsilon}\right\|}+\left((\hat{Z} \cdot Z)^{2}-1\right)^{1 / 2} \frac{X_{1} \cdot W^{\varepsilon}}{\left\|W^{\varepsilon}\right\|}
$$

which, using the triangle inequality for scalars, implies

$$
|\hat{Z} \cdot Z| \frac{\left|Z \cdot W^{\varepsilon}\right|}{\left\|W^{\varepsilon}\right\|} \leq \frac{\left|\hat{Z} \cdot W^{\varepsilon}\right|}{\left\|W^{\varepsilon}\right\|}+\left((\hat{Z} \cdot Z)^{2}-1\right)^{1 / 2} \frac{\left|X_{1} \cdot W^{\varepsilon}\right|}{\left\|W^{\varepsilon}\right\|} .
$$

Rearranging the above equation gives

$$
\frac{\left|\hat{Z} \cdot W^{\varepsilon}\right|}{\left\|W^{\varepsilon}\right\|} \geq|\hat{Z} \cdot Z| \frac{\left|Z \cdot W^{\varepsilon}\right|}{\left\|W^{\varepsilon}\right\|}-\left((\hat{Z} \cdot Z)^{2}-1\right)^{1 / 2} \frac{\left|X_{1} \cdot W^{\varepsilon}\right|}{\left\|W^{\varepsilon}\right\|}
$$

and so, using (116)

$$
\frac{\left|\hat{Z} \cdot W^{\varepsilon}\right|}{\left\|W^{\varepsilon}\right\|} \geq|\hat{Z} \cdot Z| \frac{\left|Z \cdot W^{\varepsilon}\right|}{\left\|W^{\varepsilon}\right\|}-\left((\hat{Z} \cdot Z)^{2}-1\right)^{1 / 2} \frac{\left|Z \cdot W^{\varepsilon}\right|}{\left\|W^{\varepsilon}\right\|}=\frac{\left|Z \cdot W^{\varepsilon}\right|}{\left\|W^{\varepsilon}\right\|}\left(|\hat{Z} \cdot Z|-\left((\hat{Z} \cdot Z)^{2}-1\right)^{1 / 2}\right) .
$$

Since $|\hat{Z} \cdot Z|-\left((\hat{Z} \cdot Z)^{2}-1\right)^{1 / 2}>0$, and $Z$ and $\hat{Z}$ are independent of $\varepsilon$, it follows that

$$
\lim _{\varepsilon \rightarrow 0} \frac{\left|Z \cdot W^{\varepsilon}\right|}{\left\|W^{\varepsilon}\right\|} \rightarrow \infty \Longrightarrow \lim _{\varepsilon \rightarrow 0} \frac{\left|\hat{Z} \cdot W^{\varepsilon}\right|}{\left\|W^{\varepsilon}\right\|} \rightarrow \infty .
$$

The connection between our definition of an ultra-relativistic vector field and $\mathbf{v} \cdot \mathbf{v} \simeq 1$ is exhibited by introducing the 1-parameter family of Newtonian velocities $\mathbf{v}^{\varepsilon}$ of $W^{\varepsilon}$ with respect to the observer field $U$ :

$$
\mathbf{v}^{\varepsilon}=-\frac{W^{\varepsilon}}{W^{\varepsilon} \cdot U}-U
$$

where $U \cdot U=-1$. It follows that

$$
\mathbf{v}^{\varepsilon} \cdot \mathbf{v}^{\varepsilon}=\left(\frac{W^{\varepsilon}}{W^{\varepsilon} \cdot U}+U\right) \cdot\left(\frac{W^{\varepsilon}}{W^{\varepsilon} \cdot U}+U\right)=\frac{W^{\varepsilon} \cdot W^{\varepsilon}}{\left(W^{\varepsilon} \cdot U\right)^{2}}+\frac{2\left(W^{\varepsilon} \cdot U\right)}{W^{\varepsilon} \cdot U}+U \cdot U=\frac{W^{\varepsilon} \cdot W^{\varepsilon}}{\left(W^{\varepsilon} \cdot U\right)^{2}}+1 .
$$

Thus $\lim _{\varepsilon \rightarrow 0} \mathbf{v}^{\varepsilon} \cdot \mathbf{v}^{\varepsilon}=1 \Longleftrightarrow \lim _{\varepsilon \rightarrow 0} W^{\varepsilon} \cdot W^{\varepsilon} /\left(W^{\varepsilon} \cdot U\right)^{2}=-\lim _{\varepsilon \rightarrow 0}\left(\left\|W^{\varepsilon}\right\| /\left|W^{\varepsilon} \cdot U\right|\right)^{2}=0$ and hence $\lim _{\varepsilon \rightarrow 0} \mathbf{v}^{\varepsilon} \cdot \mathbf{v}^{\varepsilon}=1 \Longleftrightarrow \lim _{\varepsilon \rightarrow 0}\left|W^{\varepsilon} \cdot U\right| /\left\|W^{\varepsilon}\right\| \rightarrow \infty$ (i.e. $W^{\varepsilon}$ is ultra-relativistic).

Observe that in definition (114) $\lim _{\varepsilon \rightarrow 0} W^{\varepsilon}$ may or may not exist. Given a 1-parameter vector field, $\mathcal{W}^{\varepsilon}, \mathcal{W}^{\varepsilon}$ is said to be light-like limited if $\mathcal{W}^{0}=\lim _{\varepsilon \rightarrow 0} \mathcal{W}^{\varepsilon}$ exists and is light-like (i.e. $\mathcal{W}^{0} \neq 0$ and $\left.\left\|\mathcal{W}^{0}\right\|=0\right)$. The relationship between ultra-relativistic vector fields and light-like limited vector fields is given by the following lemma: 
Lemma 4. If $\mathcal{W}^{\varepsilon}$ is light-like limited then $\mathcal{W}^{\varepsilon}$ is ultra-relativistic. If $\mathcal{W}^{\varepsilon}$ is ultra-relativistic and the limit $\lim _{\varepsilon \rightarrow 0} \mathcal{W}^{\varepsilon}$ exists and is nowhere 0 then $\mathcal{W}^{\varepsilon}$ is light-like limited.

Proof. If $\mathcal{W}^{\varepsilon}$ is light-like limited then $\lim _{\varepsilon \rightarrow 0}\left|\mathcal{W}^{\varepsilon} \cdot Z\right|=\left|\lim _{\varepsilon \rightarrow 0}\left(\mathcal{W}^{\varepsilon}\right) \cdot Z\right|=\left|\mathcal{W}^{0} \cdot Z\right|>0$ since $\mathcal{W}^{0}$ is light-like and $Z$ is time-like. Furthermore, $\lim _{\varepsilon \rightarrow 0}\left(\mathcal{W}^{\varepsilon} \cdot \mathcal{W}^{\varepsilon}\right)=-\left\|\mathcal{W}^{0}\right\|^{2}=0$ and so $\mathcal{W}^{\varepsilon}$ is ultra-relativistic.

If $\mathcal{W}^{\varepsilon}$ is ultra-relativistic and the limit $\mathcal{W}^{0}=\lim _{\varepsilon \rightarrow 0} \mathcal{W}^{\varepsilon}$ exists and is nowhere 0 then (since $\mathcal{W}^{0} \cdot Z$ is finite) $\left\|\mathcal{W}^{0}\right\|$ must vanish to ensure (114) is obeyed.

The 1-parameter family of velocity fields $V^{\varepsilon}$ introduced in the main body of this article (see section 3) is ultra-relativistic. Moreover, the vector fields $V_{-1}, V_{0}$ and $V_{1}$ then have certain properties:

\section{Lemma 5.}

- The 1-parameter family of vector fields $V^{\varepsilon}$ is ultra-relativistic if and only if $V_{-1}$ does not vanish anywhere.

- If $V^{\varepsilon}$ is ultra-relativistic then $V_{0}$ is nowhere time-like and $V_{1}$ does not vanish anywhere.

Proof.

- If $V_{-1} \neq 0$ then for any time-like vector $Z, V_{-1} \cdot Z \neq 0$ so

$$
\lim _{\varepsilon \rightarrow 0}\left(\left|V^{\varepsilon} \cdot Z\right| /\left\|V^{\varepsilon}\right\|\right)=\lim _{\varepsilon \rightarrow 0}\left(\varepsilon^{-1}\left|V_{-1} \cdot Z\right|\right)=\infty
$$

since $\left\|V^{\varepsilon}\right\|=1$ and so $V^{\varepsilon}$ is ultra-relativistic. If there is some $x \in \mathcal{M}$ such that $V_{-1}(x)=0$ then

$$
\lim _{\varepsilon \rightarrow 0}\left(\left|V^{\varepsilon}(x) \cdot Z(x)\right| /\left\|V^{\varepsilon}(x)\right\|\right)=\lim _{\varepsilon \rightarrow 0}\left(\varepsilon^{0}\left|V_{0}(x) \cdot Z(x)\right|\right) \neq \infty
$$

so $V^{\varepsilon}$ is not ultra-relativistic.

- Assume $V_{0}$ is time-like. Since $V^{\varepsilon}$ is ultra-relativistic it follows $V_{-1} \neq 0$ and, furthermore, since $V_{0}$ is time-like $V_{-1} \cdot V_{0} \neq 0$; however, this conclusion contradicts (90) which states $V_{0}$ and $V_{-1}$ are orthogonal. Hence, $V_{0}$ is not time-like (i.e. $V_{0}$ is space-like, light-like or zero).

Now since $V_{-1}$ is light-like and $V_{0}$ is not time-like then in order to satisfy $2 V_{-1} \cdot V_{1}+V_{0} \cdot V_{0}=-1$ from (93) $V_{1}$ does not vanish anywhere.

\section{A.2 The perturbation hierarchy in Gibbs' 3-vector notation}

To facilitate a comparison with existing fluid descriptions of beam dynamics [10, 11] that employ Gibbs' 3-vector notation the equations (52 [56) in section 3 are here transcribed into that language.

By splitting with respect to an inertial observer field $\frac{1}{c} \partial_{t}$ where

$$
g=-c^{2} d t \otimes d t+d x \otimes d x+d y \otimes d y+d z \otimes d z
$$


one obtains

$$
\begin{aligned}
& \boldsymbol{\nabla} \cdot \boldsymbol{e}=\frac{m_{0} c^{2}}{q_{0}} \gamma \rho, \\
& \boldsymbol{\nabla} \times \boldsymbol{b}=\frac{1}{q_{0}} \rho \boldsymbol{p}+\frac{1}{c^{2}} \frac{\partial \boldsymbol{e}}{\partial t}, \\
& \boldsymbol{\nabla} \times \boldsymbol{e}+\frac{\partial \boldsymbol{b}}{\partial t}=0, \\
& \boldsymbol{\nabla} \cdot \boldsymbol{b}=0 \\
& \gamma \frac{\partial \boldsymbol{p}}{\partial t}+\left(\frac{\boldsymbol{p}}{m_{0}} \cdot \boldsymbol{\nabla}\right) \boldsymbol{p}=q_{0}\left(\gamma \boldsymbol{e}+\frac{1}{m_{0}} \boldsymbol{p} \times \boldsymbol{b}\right), \\
& -\gamma^{2}+\frac{\boldsymbol{p} \cdot \boldsymbol{p}}{m_{0}^{2} c^{2}}=-1, \\
& m_{0} \frac{\partial}{\partial t}(\gamma \rho)+\nabla \cdot(\rho \boldsymbol{p})=0
\end{aligned}
$$

and

$$
\gamma \partial_{t} \gamma+\frac{\boldsymbol{p}}{m_{0}} \cdot \nabla \gamma=\frac{q_{0}}{m_{0}^{2} c^{2}} \boldsymbol{p} \cdot \boldsymbol{e}
$$

where $\boldsymbol{a} \cdot \boldsymbol{b}$ is the standard Euclidean dot product of two 3 -vector fields $\boldsymbol{a}$ and $\boldsymbol{b}$. Note that (118) can be obtained by taking the scalar product of $\boldsymbol{p}$ with the differential equation for $\boldsymbol{p}$ and the algebraic constraint on $\gamma$ and $\boldsymbol{p}$ in (117). Therefore, like the final equation in (117) (charge conservation), equation (118) is redundant. However, the two differential equations are treated very differently in the subsequent asymptotic analysis. Equation (118) is automatically maintained order-by-order by the algebraic constraint on $\gamma$ and $\boldsymbol{p}$ but charge conservation must be imposed order-by-order. Hence, (118) is not included in the following.

The scalar field $\rho$ is the reduced proper charge density introduced in the main body of the article and the MKS fields $(\boldsymbol{p}, \boldsymbol{e}, \boldsymbol{b})$ and $\gamma$ are related to $(V, F)$ by

$$
\begin{aligned}
V & =\frac{\gamma}{c} \partial_{t}+\frac{\mathbb{P}}{m_{0} c} \\
F & =\frac{q_{0}}{m_{0} c} d t \wedge \tilde{\mathbb{E}}+\frac{q_{0}}{m_{0}} \star(d t \wedge \tilde{\mathbb{B}})
\end{aligned}
$$

where $(\mathbb{P}, \mathbb{E}, \mathbb{B})$ are the vector fields

$$
\begin{aligned}
& \mathbb{P}=(\boldsymbol{p} \cdot \boldsymbol{i}) \partial_{x}+(\boldsymbol{p} \cdot \boldsymbol{j}) \partial_{y}+(\boldsymbol{p} \cdot \boldsymbol{k}) \partial_{z}, \\
& \mathbb{E}=(\boldsymbol{e} \cdot \boldsymbol{i}) \partial_{x}+(\boldsymbol{e} \cdot \boldsymbol{j}) \partial_{y}+(\boldsymbol{e} \cdot \boldsymbol{k}) \partial_{z}, \\
& \mathbb{B}=(\boldsymbol{b} \cdot \boldsymbol{i}) \partial_{x}+(\boldsymbol{b} \cdot \boldsymbol{j}) \partial_{y}+(\boldsymbol{b} \cdot \boldsymbol{k}) \partial_{z}
\end{aligned}
$$

and $(\boldsymbol{i}, \boldsymbol{j}, \boldsymbol{k})$ is an orthonormal inertial 3 -vector frame.

The expansions for the 1-parameter families $\boldsymbol{e}^{\varepsilon}, \boldsymbol{b}^{\varepsilon}, \gamma^{\varepsilon}, \boldsymbol{p}^{\varepsilon}$ and $\rho^{\varepsilon}$ corresponding to (78) are

$$
\boldsymbol{e}^{\varepsilon}=\sum_{n=-1}^{\infty} \varepsilon^{n} \boldsymbol{e}_{n}, \quad \boldsymbol{b}^{\varepsilon}=\sum_{n=-1}^{\infty} \varepsilon^{n} \boldsymbol{b}_{n}, \quad \gamma^{\varepsilon}=\sum_{n=-1}^{\infty} \varepsilon^{n} \gamma_{n}, \quad \boldsymbol{p}^{\varepsilon}=\sum_{n=-1}^{\infty} \varepsilon^{n} \boldsymbol{p}_{n}, \quad \rho^{\varepsilon}=\sum_{n=1}^{\infty} \varepsilon^{n} \rho_{n}
$$

i.e.

$$
\begin{aligned}
\boldsymbol{e}^{\varepsilon} & =\varepsilon^{-1} \boldsymbol{e}_{-1}+\boldsymbol{e}_{0}+\varepsilon \boldsymbol{e}_{1}+\ldots, & & \boldsymbol{b}^{\varepsilon}=\varepsilon^{-1} \boldsymbol{b}_{-1}+\boldsymbol{b}_{0}+\varepsilon \boldsymbol{b}_{1}+\ldots, \\
\gamma^{\varepsilon} & =\varepsilon^{-1} \gamma_{-1}+\gamma_{0}+\varepsilon \gamma_{1}+\ldots, & & \boldsymbol{p}^{\varepsilon}=\varepsilon^{-1} \boldsymbol{p}_{-1}+\boldsymbol{p}_{0}+\varepsilon \boldsymbol{p}_{1}+\ldots, \\
\rho^{\varepsilon} & =\varepsilon \rho_{1}+\varepsilon^{2} \rho_{2}+\ldots & &
\end{aligned}
$$


Substituting the above expansions into (117) yields the following hierarchy corresponding to equations (86.93):

Step 1: The source-free Maxwell equations are satisfied by the prescribed external electric field $\boldsymbol{e}_{-1}$ and external magnetic field $\boldsymbol{b}_{-1}$ :

$$
\begin{aligned}
& \boldsymbol{\nabla} \cdot \boldsymbol{e}_{-1}=0 \\
& \boldsymbol{\nabla} \times \boldsymbol{b}_{-1}=\frac{1}{c^{2}} \frac{\partial \boldsymbol{e}_{-1}}{\partial t}, \\
& \boldsymbol{\nabla} \times \boldsymbol{e}_{-1}+\frac{\partial \boldsymbol{b}_{-1}}{\partial t}=0, \\
& \boldsymbol{\nabla} \cdot \boldsymbol{b}_{-1}=0 .
\end{aligned}
$$

Step 2: The partial differential equation

$$
\gamma_{-1} \frac{\partial \boldsymbol{p}_{-1}}{\partial t}+\left(\frac{\boldsymbol{p}_{-1}}{m_{0}} \cdot \nabla\right) \boldsymbol{p}_{-1}=q_{0}\left(\gamma_{-1} \boldsymbol{e}_{-1}+\frac{1}{m_{0}} \boldsymbol{p}_{-1} \times \boldsymbol{b}_{-1}\right)
$$

and algebraic condition

$$
-\gamma_{-1}^{2}+\frac{\boldsymbol{p}_{-1} \cdot \boldsymbol{p}_{-1}}{m_{0}^{2} c^{2}}=0
$$

are solved for $\gamma_{-1}$ and $\boldsymbol{p}_{-1}$.

Step 3: The leading order equation describing charge conservation is

$$
m_{0} \frac{\partial}{\partial t}\left(\gamma_{-1} \rho_{1}\right)+\nabla \cdot\left(\rho_{1} \boldsymbol{p}_{-1}\right)=0
$$

and is solved for $\rho_{1}$.

Step 4: Solve the Maxwell equations for $\boldsymbol{e}_{0}$ and $\boldsymbol{b}_{0}$ with $\frac{m_{0} c^{2}}{q_{0}} \gamma_{-1} \rho_{1}$ and $\frac{1}{q_{0}} \rho_{1} \boldsymbol{p}_{-1}$ as sources:

$$
\begin{aligned}
& \boldsymbol{\nabla} \cdot \boldsymbol{e}_{0}=\frac{m_{0} c^{2}}{q_{0}} \gamma_{-1} \rho_{1}, \\
& \boldsymbol{\nabla} \times \boldsymbol{b}_{0}=\frac{1}{q_{0}} \rho_{1} \boldsymbol{p}_{-1}+\frac{1}{c^{2}} \frac{\partial \boldsymbol{e}_{0}}{\partial t}, \\
& \nabla \times \boldsymbol{e}_{0}+\frac{\partial \boldsymbol{b}_{0}}{\partial t}=0, \\
& \boldsymbol{\nabla} \cdot \boldsymbol{b}_{0}=0 .
\end{aligned}
$$

Step 5: Solve

$$
\begin{aligned}
\gamma_{-1} \frac{\partial \boldsymbol{p}_{0}}{\partial t}+\gamma_{0} \frac{\partial \boldsymbol{p}_{-1}}{\partial t} & +\left(\frac{\boldsymbol{p}_{0}}{m_{0}} \cdot \boldsymbol{\nabla}\right) \boldsymbol{p}_{-1}+\left(\frac{\boldsymbol{p}_{-1}}{m_{0}} \cdot \nabla\right) \boldsymbol{p}_{0} \\
& =q_{0}\left(\gamma_{-1} \boldsymbol{e}_{0}+\gamma_{0} \boldsymbol{e}_{-1}\right)+\frac{q_{0}}{m_{0}}\left(\boldsymbol{p}_{-1} \times \boldsymbol{b}_{0}+\boldsymbol{p}_{0} \times \boldsymbol{b}_{-1}\right), \\
-\gamma_{-1} \gamma_{0}+\frac{\boldsymbol{p}_{-1} \cdot \boldsymbol{p}_{0}}{m_{0}^{2} c^{2}} & =0
\end{aligned}
$$

for $\gamma_{0}$ and $\boldsymbol{p}_{0}$. Use of the above algebraic equation to eliminate $\gamma_{0}$ leads to an inhomogeneous linear partial differential equation for $\boldsymbol{p}_{0}$. 
Step 6: The equation

$$
m_{0} \frac{\partial}{\partial t}\left(\gamma_{0} \rho_{1}\right)+m_{0} \frac{\partial}{\partial t}\left(\gamma_{-1} \rho_{2}\right)+\nabla \cdot\left(\rho_{1} \boldsymbol{p}_{0}\right)+\nabla \cdot\left(\rho_{2} \boldsymbol{p}_{-1}\right)=0
$$

enforcing charge conservation is solved for $\rho_{2}$.

Step 7: The electric and magnetic fields $\boldsymbol{e}_{1}$ and $\boldsymbol{b}_{1}$ are solutions to the following Maxwell equations:

$$
\begin{aligned}
& \boldsymbol{\nabla} \cdot \boldsymbol{e}_{1}=\frac{m_{0} c^{2}}{q_{0}}\left(\gamma_{-1} \rho_{2}+\gamma_{0} \rho_{1}\right), \\
& \boldsymbol{\nabla} \times \boldsymbol{b}_{1}=\frac{1}{q_{0}}\left(\rho_{2} \boldsymbol{p}_{-1}+\rho_{1} \boldsymbol{p}_{0}\right)+\frac{1}{c^{2}} \frac{\partial \boldsymbol{e}_{1}}{\partial t} \\
& \boldsymbol{\nabla} \times \boldsymbol{e}_{1}+\frac{\partial \boldsymbol{b}_{1}}{\partial t}=0 \\
& \boldsymbol{\nabla} \cdot \boldsymbol{b}_{1}=0 .
\end{aligned}
$$

Step 8: Solve

$$
\begin{aligned}
\gamma_{-1} \frac{\partial \boldsymbol{p}_{1}}{\partial t}+\gamma_{0} \frac{\partial \boldsymbol{p}_{0}}{\partial t}+ & \gamma_{1} \frac{\partial \boldsymbol{p}_{-1}}{\partial t}+\left(\frac{\boldsymbol{p}_{-1}}{m_{0}} \cdot \boldsymbol{\nabla}\right) \boldsymbol{p}_{1}+\left(\frac{\boldsymbol{p}_{0}}{m_{0}} \cdot \nabla\right) \boldsymbol{p}_{0}+\left(\frac{\boldsymbol{p}_{1}}{m_{0}} \cdot \boldsymbol{\nabla}\right) \boldsymbol{p}_{-1} \\
& =q_{0}\left(\gamma_{-1} \boldsymbol{e}_{1}+\gamma_{0} \boldsymbol{e}_{0}+\gamma_{1} \boldsymbol{e}_{-1}\right)+\frac{q_{0}}{m_{0}}\left(\boldsymbol{p}_{-1} \times \boldsymbol{b}_{1}+\boldsymbol{p}_{0} \times \boldsymbol{b}_{0}+\boldsymbol{p}_{1} \times \boldsymbol{b}_{-1}\right)
\end{aligned}
$$

for $\gamma_{1}$ and $\boldsymbol{p}_{1}$. Eliminating $\gamma_{1}$ using the above algebraic equation leads to an inhomogeneous linear partial differential equation for $\boldsymbol{p}_{1}$. 